\title{
7 Nematostella vectensis as a Model System
}

\author{
Layla Al-Shaer, Jamie Havrilak and Michael J. Layden
}

\section{CONTENTS}

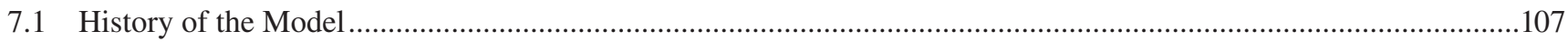

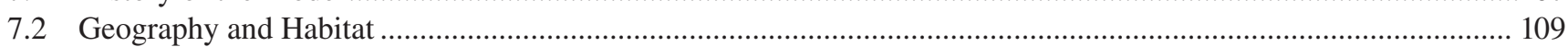

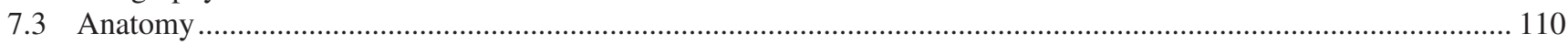

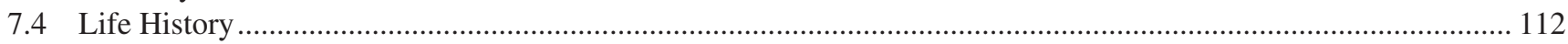

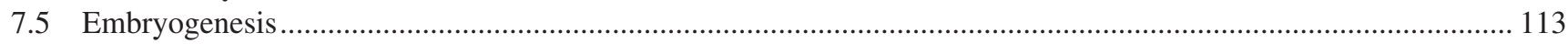

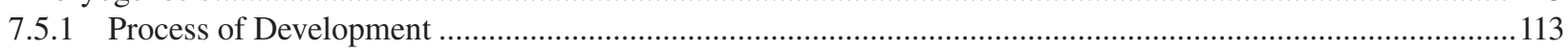

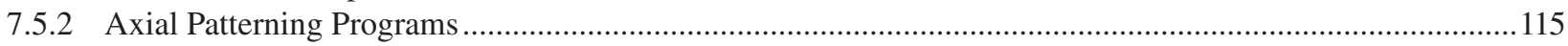

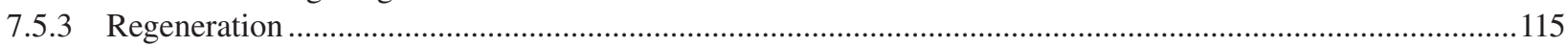

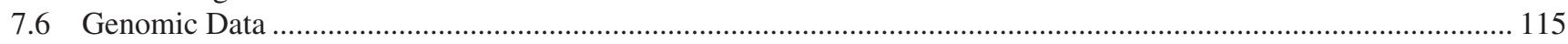

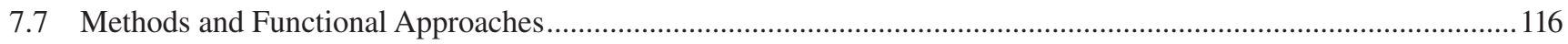

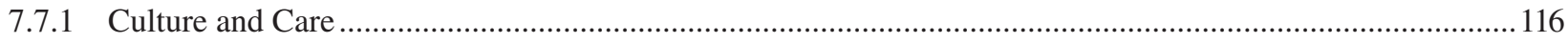

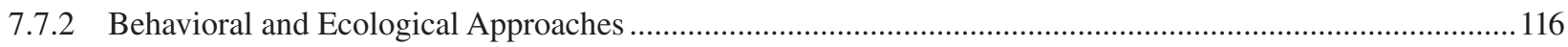

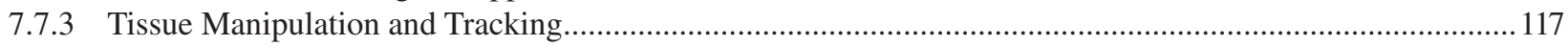

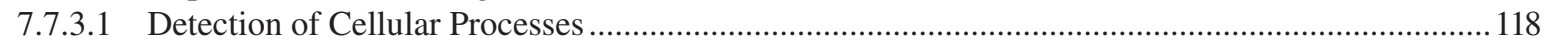

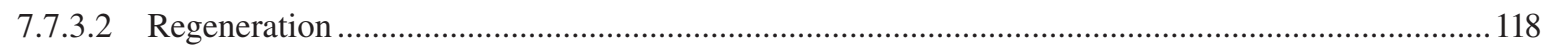

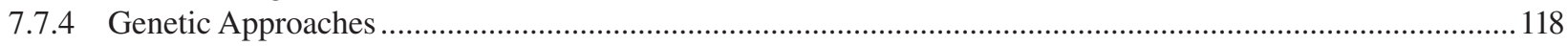

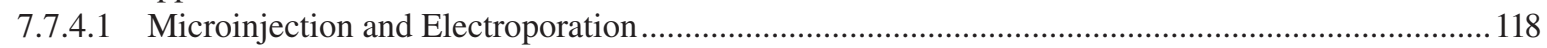

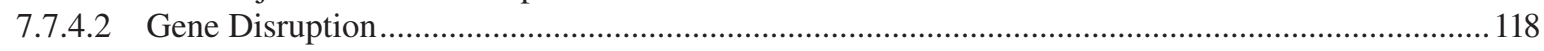

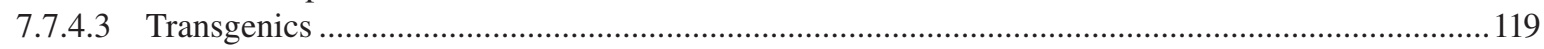

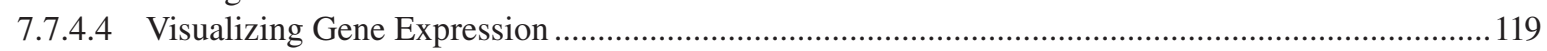

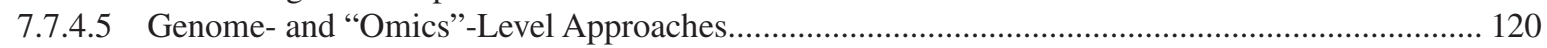

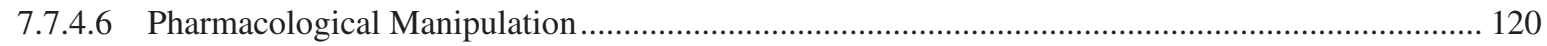

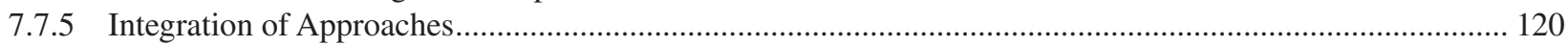

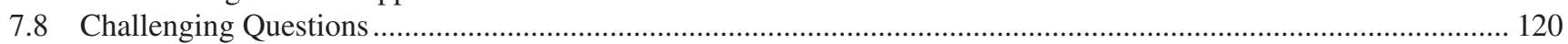

7.8.1 Is There a Deep Evolutionary Origin for Key Bilaterian Traits? .......................................................... 120

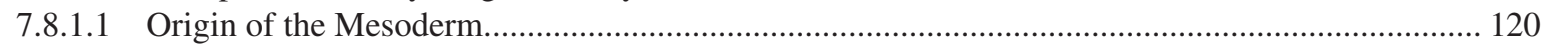

7.8.1.2 Mechanisms of Axial Patterning Leading to Bilaterality..............................................................121

7.8.2 Can Nematostella Be Used as a Cnidarian Model for Cnidarians? .............................................................. 122

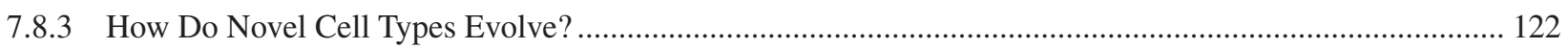

7.8.4 Does Regeneration Recapitulate Development?................................................................................. 123

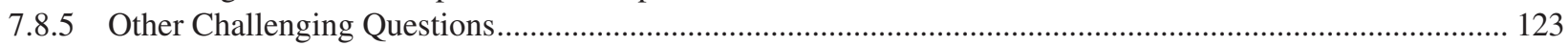

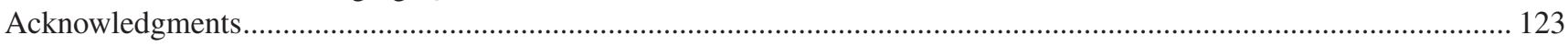

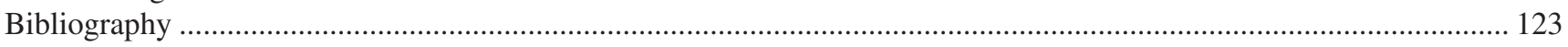

\subsection{HISTORY OF THE MODEL}

Nematostella vectensis (the starlet sea anemone) are anthozoan cnidarians. Anthozoans (e.g. corals, anemones) derive their name from the Greek anthos-flower-and zoiaanimals-because their dominant polyp form shared by this class represents "a highly colored and many-petaled flower" (Figure 7.1a) (Gosse 1860). Additionally, the different morphological states of the animal can be described as the "flower" (anthus), when all the tentacles are extended, or the "button" (oncus), when the tentacles are retracted and the oral end closes in around them (Gosse 1860).

The first description of Nematostella vectensis was published in 1935 by Thomas A. Stephenson. Stephenson attributed the discovery of Nematostella vectensis and observations of their nematosomes to Ms. Gertrude F. Selwood. She found them at the Isle of Wight (England) (Figures 7.1b, 7.2) in 1929 when she was a lecturer at Municipal College, Portsmith, and sent specimens to Stephenson. Stephenson described the free-swimming nematosomes in the gastric 

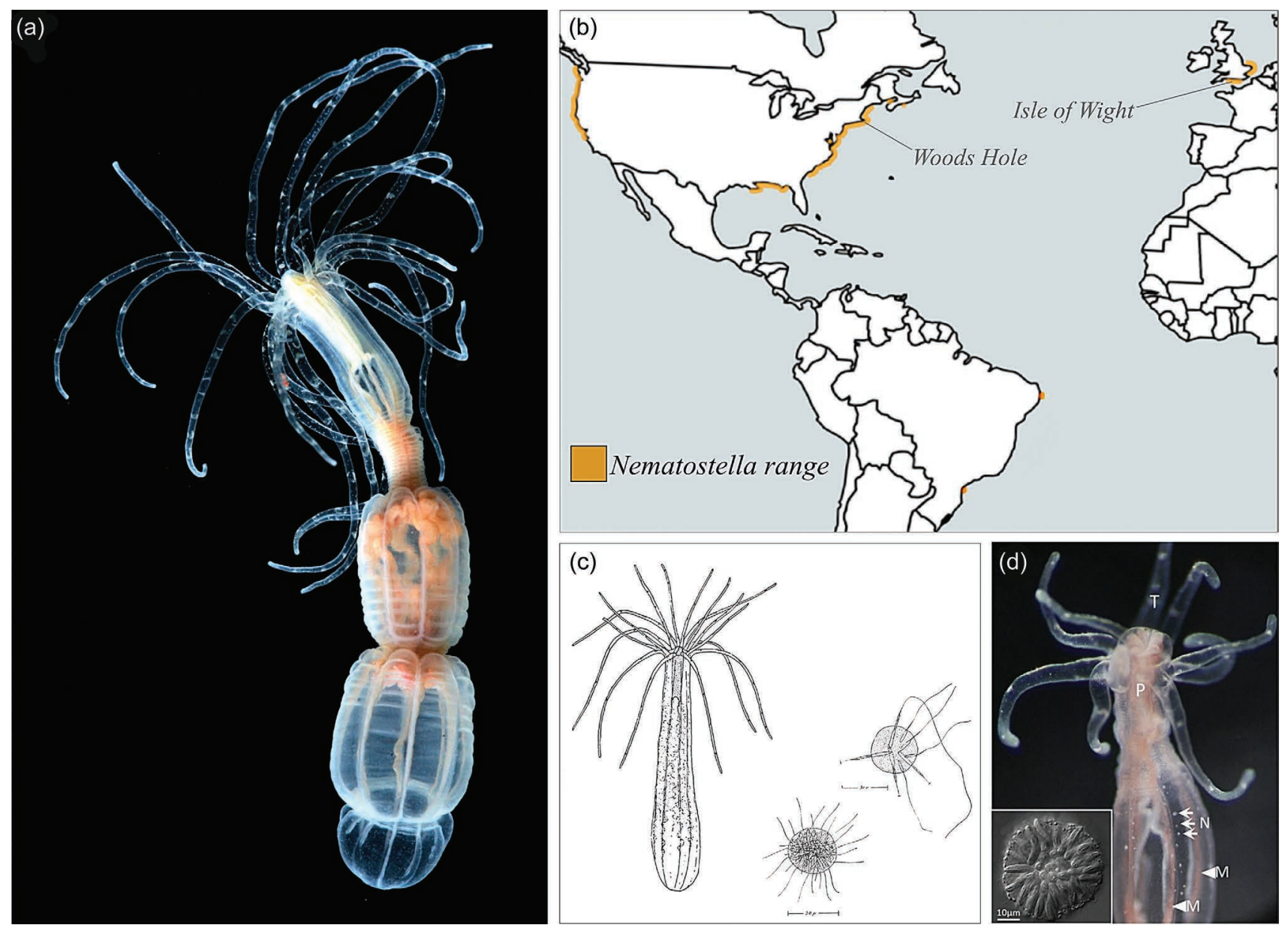

FIGURE 7.1 Characteristics and geographical range of Nematostella vectensis. (a) An adult polyp, image courtesy of Eric Röttinger. (b) Known geographical range. (c) First known illustrations of a Nematostella polyp and nematosomes with intact (left) and fired (right) cnidocytes. (d) Polyp showing tentacles (T) pharynx (P), mesenteries (M) and nematosomes (N). ([b] Illustrations by Sears Crowell 1946; [d] image modified from Babonis et al. 2016.)

cavity that became the characteristic feature of Nematostella (Figure 7.1c,d) (Stephenson 1935).

In 1939, William J. Bowden discovered Nematostella pellucida at Woods Hole, Massachusetts (Figure 7.1b), which was later described and published by Sears Crowell in 1946 (Figure 7.1c). Nematostella pellucida was initially considered distinct from vectensis due to color patterns on the body and the large geographical separation (Williams 1975; Williams 1976). Crowell suspected N. vectensis and N. pellucida were synonymous species, but because of the war, he was unable to get hold of Nematostella from the British Isles for direct comparisons (Crowell 1946). In 1957, Cadet Hand compared anemones from America and England and determined they were both Nematostella vectensis (Figure 7.2) (Hand 1957; Williams 1975; Williams 1976).

The various life history stages of Nematostella were described by several different groups from the 1940s to the 1980s (summarized in Hand and Uhlinger 1992). The potential for Nematostella as a laboratory model came when Cadet Hand and Kevin Uhlinger documented the ease of its culturability in the early 1990s (1992). Its ability to tolerate wide variations in salinity and temperature made it easy to maintain in laboratory cultures (Williams 1975; Williams 1976). Perhaps most importantly, Nematostella spawn readily with increased temperature and light. Early work established various environmental conditions that influence oogenesis, such as nutrient amount, temperature, light, density of sperm and the ideal timeframe for fertilization (Hand and Uhlinger 1992; Fritzenwanker and Technau 2002). The ability to reliably obtain thousands of embryos per spawn and close the life cycle in culture made Nematostella stand out as a potential cnidarian model system. Plus, due to its phylogenetic position as a basal metazoan, it is also especially well suited for evolutionary and developmental biology (evo-devo) studies.

Through the 1990s and early 2000s, studies focused on identifying the expression of known bilaterian homologues during Nematostella development (Figure 7.2). Initial studies focused on genes involved in axial patterning and triploblasty and provided initial insights into the origin and evolution of these genes and thus the bilaterian traits they regulate (see Darling et al. 2005). Similarly, extensive efforts 


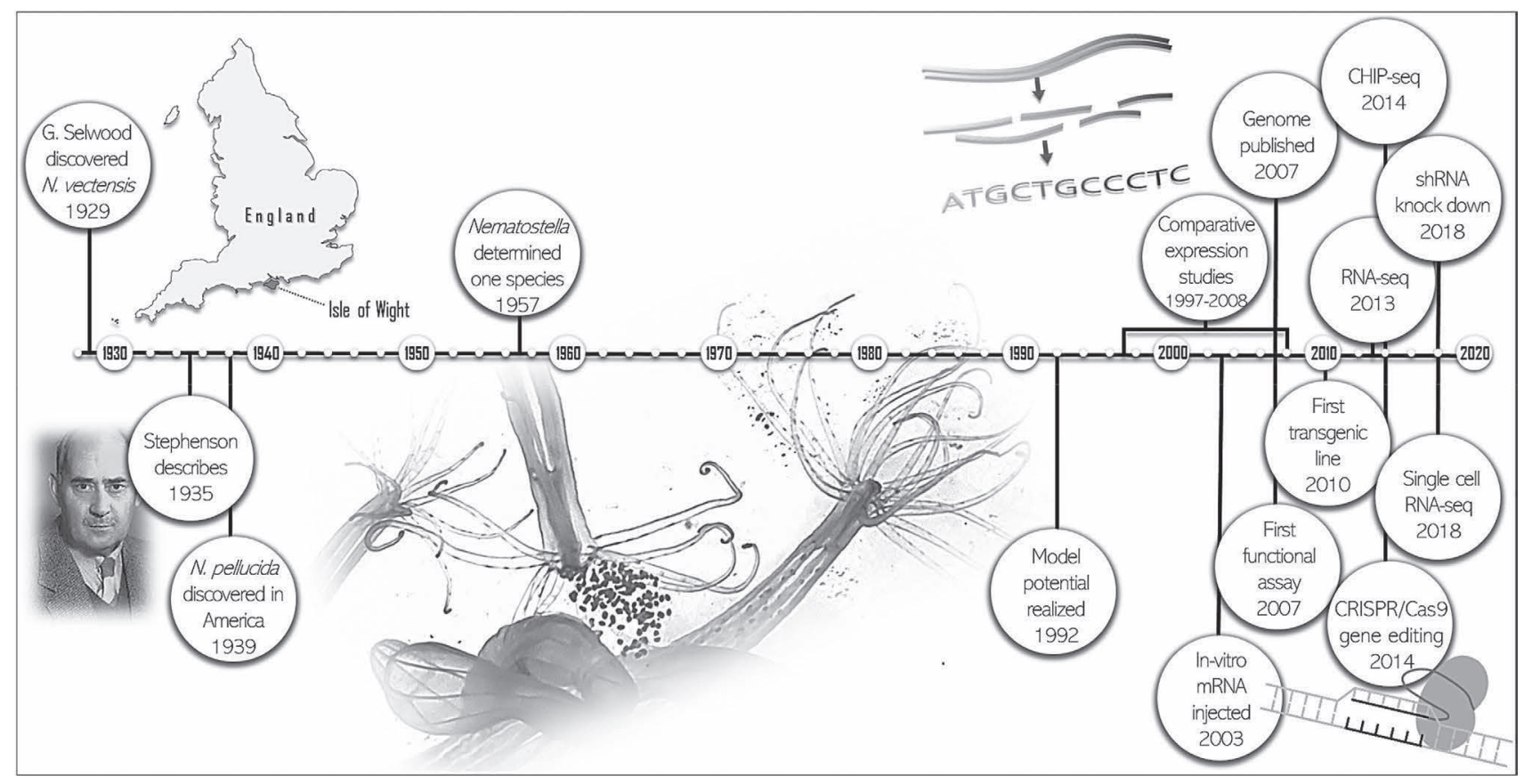

FIGURE 7.2 A timeline of major events in the Nematostella model system. (Picture of TA Stephenson adapted from Yonge 1962.)

focused on identifying expression of deeply conserved developmental signaling cascades. Comparative genomic studies have identified that genes involved in major families and signaling cascades were all present in the urbilaterian ancestor (e.g. Kortschak et al. 2003; Magie et al. 2005; Putnam et al. 2007). Furthermore, many cnidarian amino acid sequences are more like vertebrate sequences than other common model systems (Kortschak et al. 2003; Putnam et al. 2007) which supported the need to develop Nematostella as another model through the transition to more functional studies.

The application of molecular tools and reverse genetic approaches fueled the growth and use of Nematostella (Figure 7.2). With advances in sequencing technology and the publication of the genome in 2007 (Putnam et al. 2007), there has been a rapid increase in usage of Nematostella as a model organism. Morpholinos were first used successfully in Nematostella in 2005, and the first morphant phenotype was reported in 2008 (Magie et al. 2005; Rentzsch et al. 2008). Together these findings fueled the growth of functional studies, which have grown to include additional methods of gene knockdown and misexpression. The first transgenic line was published in 2010 with the creation of a muscle-specific reporter line, and transgenic reporter animals have also been used to identify and track specific cell types (Layden et al. 2012; Nakanishi et al. 2012).

Nematostella has repeatedly shown that it is amenable to novel and state-of-the-art molecular techniques. Genomiclevel analyses were established for microarray, ChIP-seq and RNA-seq (Röttinger et al. 2012; Fritz et al. 2013; Helm et al. 2013; Tulin et al. 2013; Schwaiger et al. 2014; Sinigaglia et al. 2015). Cellular dissociation protocols and advances in single-cell sequencing technology have been successfully applied, and the use of single-cell RNA-sequencing has allowed for interrogation of the complexity of Nematostella cell types and characterization of gene regulatory programs (Sebé-Pedrós et al. 2018).

Their transparent body, relatively "simple" body plan, external fertilization, ease of embryo manipulation and closed life cycle in the lab make Nematostella amenable to a myriad of research approaches and questions, including the ability to compare development and regeneration. The application of next-generation approaches has cemented the use of Nematostella as a model organism. Nematostella joins several other cnidarian species that have become more commonly utilized laboratory models, such as Hydractinia and Clytia. Hydra have long been established as a model for regeneration, but they are not as amenable to developmental studies. The combination of knowledge gained from multiple cnidarian species will help to understand the ancestral toolkit in the common ancestor that gave rise to both the cnidarian and bilaterian lineages.

\subsection{GEOGRAPHY AND HABITAT}

Native to the Atlantic coast of North America (Hand and Uhlinger 1995; Reitzel et al. 2007), the geographic range of Nematostella has expanded through anthropogenic introduction to locations across at least three continents. In North America, abundant populations have been observed along the Atlantic coast from Nova Scotia to Georgia, along the Pacific coast from Washington to California and along the Gulf coast from Florida to Louisiana (Hand and Uhlinger 1992; Hand and Uhlinger 1994). In Europe, Nematostella occur in limited number in locations along the southern and 
eastern coasts of England (Stephenson 1935; Sheader et al. 1997; Pearson et al. 2002), and in South America, where they have been found in locales off the coast of Brazil (Figure 7.1b) (Silva et al. 2010; Brandão et al. 2019). Genetic and phylogeographical analyses indicate that global populations are isolated and therefore unlikely to have spread via natural dispersal mechanisms. (Hand and Uhlinger 1995; Reitzel et al. 2007). More plausible is that they were carried as hitchhikers in the ballasts of commercial seafood vessels, creating the potential for new populations to become established outside of the natural range (Sheader et al. 1997; Takahashi et al. 2008).

The successful geographic expansion of Nematostella can likely be attributed to their environmental plasticity, as they can inhabit a variety of coastal habitats and can tolerate fluctuating environmental conditions. They often occur burrowed in soft muddy sediments of poikilohaline lagoons, brackish mudflats, salt marshes and creeks and subtidal areas of certain estuaries and bays (Williams 1975; Williams 1976). As eurythermal animals, they can survive and adapt to a wide range of temperatures and have even been found living in habitats that approach their physiological upper limit of approximately $40^{\circ} \mathrm{C}$ (Williams 1975 ; Williams 1976). As euryhaline animals, they can contend with the spatiotemporal fluctuations in salinity common in the estuarine habitats where they are found. A testament to the remarkably flexible physiology of this anemone is that both asexual and sexual reproduction can occur under a wide range of salinities (Hand and Uhlinger 1992).

\subsection{ANATOMY}

Adult Nematostella are transparent and possess the classic polyp morphology found throughout the cnidarian lineage (Figure 7.3a). Atop the oral end of the body column is an opening that is surrounded by 4-18 long stinging tentacles, which aid in prey capture and defense but also expand the surface area of the gastric cavity (Fritz et al. 2013; Ikmi et al. 2020). This oral opening serves as both a mouth and anus by attaching to a blind-ended gut through a noticeable pharynx (Williams 1975; Williams 1976). There is also a small pore at the aboral pole (Amiel et al. 2015). The oralaboral axis is elongated, which gives the body column a tube-like structure. Eight radially repeating body segments, which are centered around the long oral-aboral axis, give the animal what appears to be an octoradial symmetry (Figure 7.3a).

Cnidarians are generally classified as having a radially symmetric body plan, but many species have subtle bilateral differences in their anatomy that are superimposed over a general radial body plan (Martindale et al. 2002). These bilateral differences point to the presence of a secondary directive axis, which runs perpendicular to the primary oralaboral axis (Figure 7.3b) (Berking 2007). In Nematostella, the presence of a directive axis is morphologically evident in adult polyps from the slit-like shape of the oral opening and pharynx, the presence of a ciliated groove (siphonoglyph) on one side of the pharynx, the asymmetric arrangement of the retractor muscles within the mesenteries and the asymmetric arrangement of the tentacles around the oral opening (Martindale et al. 2002; Berking 2007; He et al. 2018).

Nematostella are diploblastic, meaning that the entire body is composed of cells derived from two germ layers: an outer ectoderm which forms the epidermis and a bifunctional internal endoderm which forms the gastrodermis (Figure 7.3a) (Finnerty et al. 2004; Wijesena et al. 2017; but see Steinmetz et al. 2017). The epidermis covers the outside of the animal and serves as a protective barrier between the animal and its environment, while the bifunctional gastrodermis lines the coelenteron and provides both absorptive and contractile functions (Martindale et al. 2004). Separating the ectoderm and endoderm is the mesoglea, a thin extracellular matrix with no organized tissue and only a few migratory amoebocyte cells of unknown function (Tucker et al. 2011). The ectoderm contains primarily columnar cell epithelia (Magie et al. 2007), along with other differentiated cells, including stinging cells called cnidocytes (Frank and Bleakney 1976), sensory neurons, ganglion neurons (Marlow et al. 2009; Sinigaglia et al. 2015; Leclère et al. 2016), a population of myoepithelial (muscle) cells in the tentacles (Jahnel et al. 2014) and gland cells (Frank and Bleakney 1976). Ectodermal gland cells include those with exocrine and insulinergic functions (Steinmetz et al. 2017), and some produce a potent neurotoxin for both prey capture and defense (Moran et al. 2011). The endoderm possesses squamous epithelial cells (Magie et al. 2007), sensory and ganglion neurons (Marlow et al. 2009; Sinigaglia et al. 2015; Leclère et al. 2016), the majority of myoepithelial cells (Jahnel et al. 2014), gland cells (Frank and Bleakney 1976; Steinmetz et al. 2017) and gametic and absorptive cells (Layden et al. 2012; Nakanishi et al. 2012). This basic organization results in epithelial cells and differentiated cell types being scattered and intermixed with one another, as opposed to being organized into discrete organ systems.

Apart from the pharynx, the most obvious internal structures of adult Nematostella are the ecto- and endodermally derived lamellae known as mesenteries (Steinmetz et al. 2017). Adults have eight mesenteries, one in each body segment, that look ruffled in appearance and run the length of the body column (Figure 7.3a). Each mesentery arises from the pharynx and consists of two layers of gastrodermis epithelium separated by a layer of mesoglea (Martindale et al. 2004). Structurally, the mesenteries are important because they provide support for the pharynx, they contain muscles that allow for quick contractions of the body column (Renfer et al. 2010) and they increase the surface area of the gastrodermis. Physiologically, the mesenteries are incredibly multifunctional, as they contain absorptive cells that aid in digestion and nutrient uptake and are where gametes (Martindale et al. 2004), cnidocytes (Steinmetz 2019) and nematosomes are produced (Williams 1975, 1976). Nematosomes are the defining apomorphy of Nematostella (Williams 1975, 1976, 1979). They are multicellular, spherical, flagellated bodies that contain cnidocytes and can be 


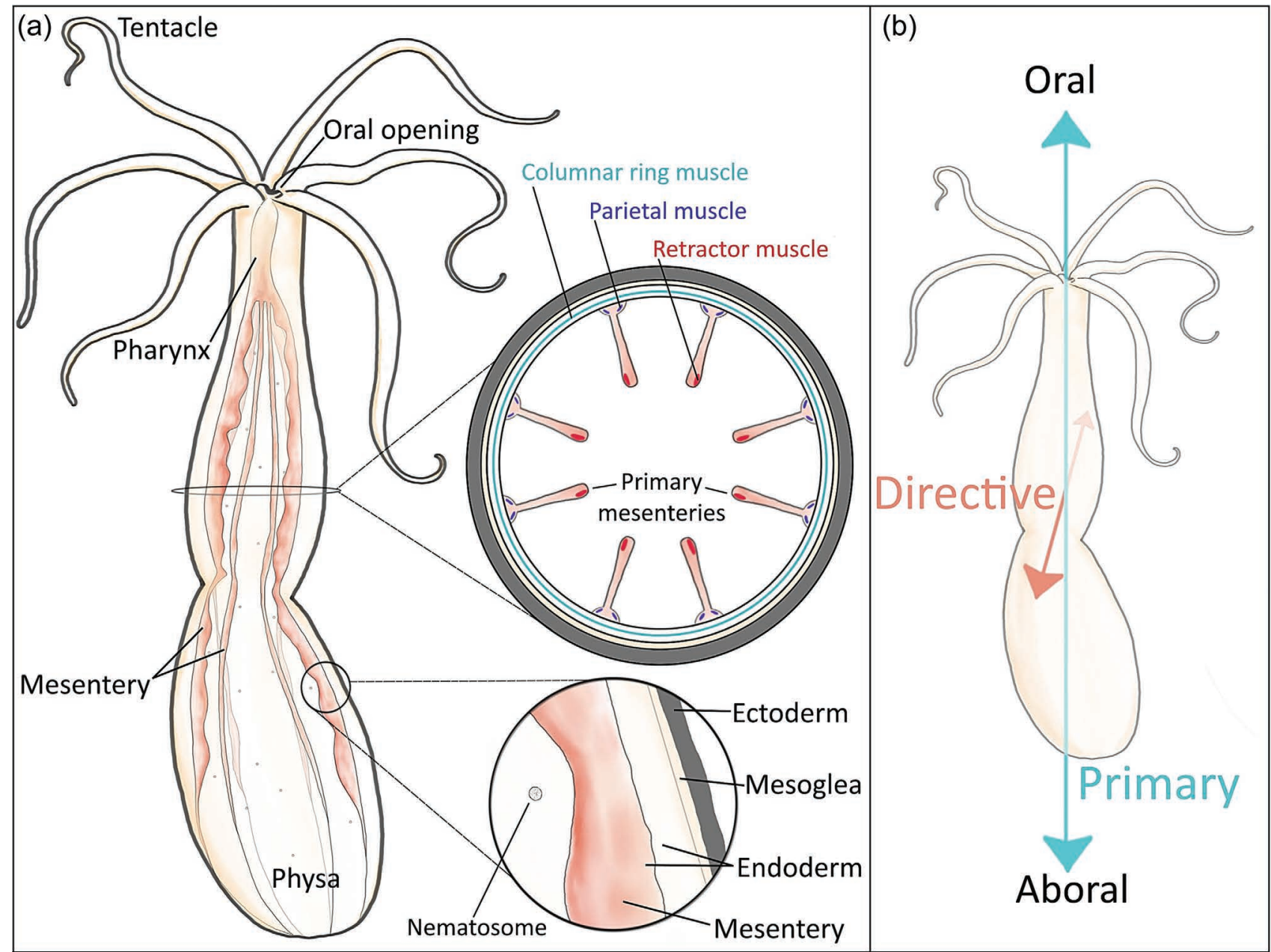

(c)

FIGURE 7.3 Anatomy of Nematostella. (a) Schematic of an adult polyp. (b) The primary oral-aboral axis is orthogonal to the secondary directive axis. (c) A transgenic animal is used to visualize a subset of neurons in the nerve net. Arrowheads show longitudinal neuronal tracts.

found in abundance throughout the coelenteron and packaged into egg masses (Williams 1975, 1976).

Nematostella have five functionally and morphologically distinct myoepithelial cell groups that together make up the body column and tentacular muscle systems. In the body column there are three muscle groups (Figure 7.3a). The longitudinally oriented parietal and retractor muscles are found within different regions of each mesentery and 
run the length of the oral-aboral axis. The columnar ring muscle group wraps around the circumference of the body wall along the oral-aboral axis (Jahnel et al. 2014). The tentacles have a similar muscle system; they contain longitudinal muscles that run the length of each tentacle, as well as ring muscles that are oriented orthogonally to the tentacular longitudinal muscles.

Nematostella possess a nerve-net nervous system-aptly named due to the way that the neurites extend from neural soma to form a diffuse interconnected web around the organism (Figure 7.3c). The nervous system is composed of both ectodermal and endodermal nerve nets (Layden et al. 2012; Nakanishi et al. 2012). Although they lack a centralized nervous system, there are distinct neural structures, including bundles of neurons that flank each mesentery within a longitudinal tract (Figure 7.3c) and condensations of neurons forming "nerve rings" around the oral opening and pharynx (Marlow et al. 2009; Sinigaglia et al. 2015; Leclère et al. 2016).

Neural cell types fall under three categories and can be found intermixed among other cell types. In the ectoderm, neural progenitor cells give rise to epithelial sensory cells (which extend an apical cilium to the body surface and neuronal processes basally) and ganglion cells (which lose their apical contacts and migrate so that their cell bodies are basally situated) (Marlow et al. 2009; Sinigaglia et al. 2015; Leclère et al. 2016). Unlike the sensory cells in the ectoderm, those in the endoderm lose their elongated appearance and become shortened along the apical-basal axis (Nakanishi et al. 2012). Cnidocytes are also considered nerve cells due to their neurophysiological properties, structure and calcium/mechanosensory-dependent exocytosis (Kass-Simon and Scappaticci 2002; Thurm et al. 2004; Galliot et al. 2009). Cnidocytes contain a unique organelle called a cnidocyst, which consists of a capsule and a harpoon-like structure that can be fired at ultra-fast speeds (Szczepanek et al. 2002). Capsules are highly specialized based on their function (e.g. feeding, defense, locomotion) and are classified based on their structure (Kass-Simon and Scappaticci 2002). Nematostella have three types of cnidocytes: spirocytes and two types of nematocytes. Spirocytes contain spirocyst capsules, which lack a shaft and barbs, and are found in the tentacles. Nematocytes with microbasic p-mastigophore capsules are found in the mesenteries and pharynx, and nematocytes with different-sized basitrichous isorhiza capsules are found mostly in the body wall, but also in the tentacles, mesenteries and pharynx (Williams 1975; Williams 1976).

Based on molecular and morphological observations, the three neural groups likely contain many subtypes that can be distinguished based on attributes including neurite number, neuropeptide profile, morphology and location (Nakanishi et al. 2012; Havrilak et al. 2017; Zang and Nakanishi 2020). Although the nerve net of Nematostella has been previously described as random because of its disorganized appearance (Hejnol and Rentzsch 2015), there is growing evidence that the nerve net is specifically patterned. The identification of specific neural subtypes points to a previously underappreciated complexity within the nervous system of Nematostella, and the presence of large neural structures suggests that neurogenesis is not random. In fact, it has also been shown that several specific neural subtypes exhibit a stereotyped developmental pattern (Havrilak et al. 2017).

\subsection{LIFE HISTORY}

Nematostella is a dioecious species that sexually reproduces by external fertilization, synchronously releasing eggs and sperm into the water column (Figure 7.4a) (Hand and Uhlinger 1992). Females release egg masses inside of a gelatinous sac containing nematosomes (Figure 7.4b), which are thought to provide defense to the embryos (Babonis et al. 2016). However, this does not make them immune to all predation; for example, grass shrimp will consume Nematostella embryos (Columbus-Shenkar et al. 2018). Embryos emerge from the protective sac as spherical, ciliated, non-feeding, planula larvae $\sim 36-48$ hours postfertilization (Hand and Uhlinger 1992). The free-swimming planulae elongate before metamorphosing into sessile primary polyps. Metamorphosis occurs roughly six days postfertilization and is characterized by the development of an oral opening surrounded by tentacle buds, the first two mesenteries and a loss of swimming ability that leads to larval settlement (Hand and Uhlinger 1992; Fritzenwanker et al. 2007; Fritz et al. 2013). Once settled, juvenile polyps begin to grow and mature in a nutrient-dependent manner ( Ikmi et al. 2020). The polyps are opportunistic predators that feed on small estuarine invertebrates captured by their stinging tentacles (Frank and Bleakney 1978; Posey and Hines 1991). Polyps are infaunal, preferring to burrow their body column into soft substrate so that only the oral opening and tentacles are exposed (although they are sometimes found attached to vegetation) (Williams 1975; Williams 1976). Burrowing helps to protect the body column from predation and forces would-be predators to contend with their stinging tentacles first (Columbus-Shenkar et al. 2018). Sexually mature adults range in size and will grow and shrink in response to nutrient availability (Hand and Uhlinger 1994; Havrilak et al. 2021). This phenotypic plasticity allows animals to easily adapt to environmental changes and suggests that there is no set size state (Havrilak et al. 2021). In the wild, adults are typically a few centimeters in length and will reach sexual maturity in approximately six months or less (Williams 1983). In culture, this can occur in as little as ten weeks for well-fed animals (Hand and Uhlinger 1992).

Adult Nematostella also reproduce asexually by generating clonal individuals through two forms of transverse fission: physal pinching and polarity reversal. Physal pinching is facilitated by a deep, sustained, constriction of a site along the posterior end of the body column (physa) and results in the separation of the smaller physal fragment from the rest of the anemone (Figure 7.4a). After a few days of separation, the physal fragment will begin to generate oral structures and tentacles that will allow it to feed, ultimately resulting 
in a functional clone (Hand and Uhlinger 1995; Reitzel et al. 2007). Although frequent feeding can increase the amount of transverse fission that will occur in a population, there is no correlation between parent size and the size of the physal fragments they produce. Further, the number of clones generated by individuals is highly variable; in a sibling population, some will produce several clones, while others will produce none (Hand and Uhlinger 1995). Polarity reversal is like physal pinching, except that the sequence of events is different. With polarity reversal, an adult first manifests oral structures and tentacles at the aboral end of the body column, replacing the physa. A new physa will develop midway along the body column, and physal pinching will act to separate the animal into two individuals (Reitzel et al. 2007).

It is unclear which, if any, environmental conditions promote sexual versus asexual reproduction. Since Nematostella maintain multiple modes of reproduction, it is assumed that specific environmental and/or genetic conditions exist under which each mode would have a fitness benefit. Nematostella is one of only a handful of anemone species to have multiple modes of asexual reproduction (Reitzel et al. 2007), and asexual reproduction by transverse fission is rare among anthozoan cnidarians (Fautin 2002). In Nematostella, transverse fission by polarity reversal is less common than physal pinching and may rely on seasonal environmental cues (Frank and Bleakney 1978; Reitzel et al. 2007).

Nematostella is highly regenerative, capable of bidirectional whole body-axis regeneration and regeneration of specific structures. Although regeneration following bisection is reminiscent of physal pinching, it is markedly different

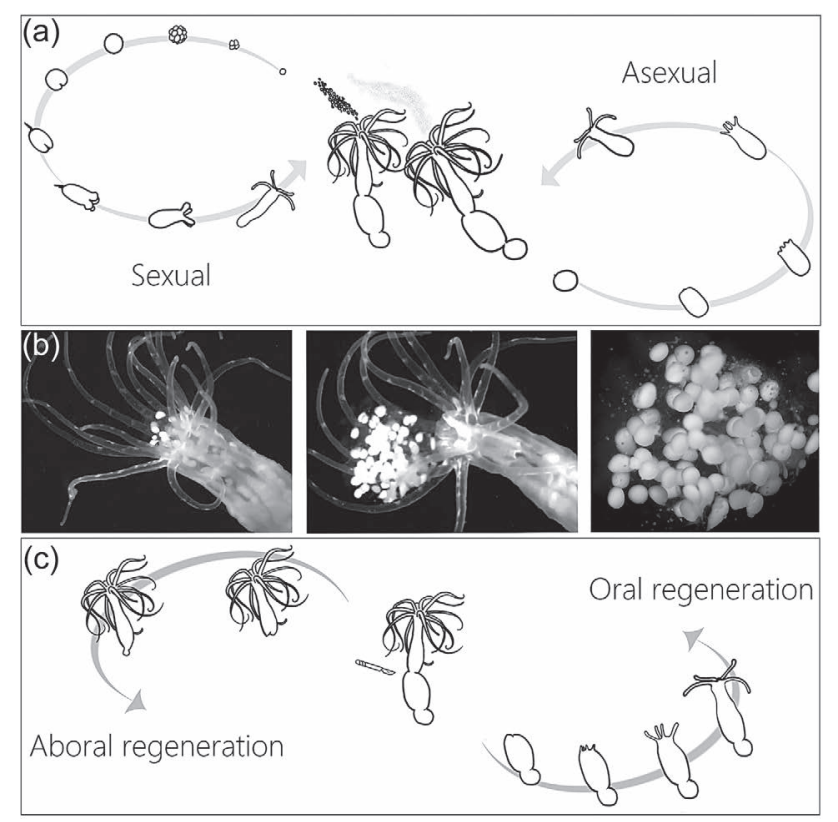

FIGURE 7.4 Reproduction and regeneration in Nematostella. (a) Sexual reproduction and asexual reproduction by physal pinching. (b) Spawning female releasing a clutch of eggs through the oral opening. Right panel shows the eggs shortly after being released. (c) Regeneration of oral and aboral fragments following wholebody axis bisection. because it is caused by an external factor that wounds the animal as opposed to an endogenously triggered constriction of the body column. When a complete bisection of the body column into oral and aboral fragments occurs, both fragments will regenerate missing structures, leading to the generation of two clonal individuals $\sim$ six to seven days postamputation (Figure 7.4c) (Reitzel et al. 2007; Amiel et al. 2015; Havrilak et al. 2021).

\subsection{EMBRYOGENESIS}

\subsubsection{Process of Development}

Embryogenesis can be investigated in its entirety since males and females release gametes into the water column and fertilization occurs externally (Figure 7.5). Zygote to juvenile polyp typically requires $\sim \operatorname{seven~days~at~} 22^{\circ} \mathrm{C}$. However, development is temperature dependent and can be sped up or slowed down by increasing or decreasing temperatures, respectively. The first cleavage initiates $\sim$ two hours after fertilization. The first two cleavage furrows typically originate perpendicular to one another at the animal pole and progress toward the vegetal pole. Initially, cytokinesis is incomplete, and it is not until the 8-cell stage that the blastomeres become separated. While a clutch of embryos will have relatively synchronous development, there is some variability of early cleavage patterns, and odd numbers of blastomeres are occasionally observed (Fritzenwanker et al. 2007, Reitzel et al. 2007). From the 16-cell stage and on, most embryos look similar, and the blastomeres are roughly similar in size (Figure 7.5b). The blastocoel becomes visible by six hours post-fertilization, following epithelialization (Figure 7.5c), which occurs between the 16- and 32-cell stages (Fritzenwanker et al. 2007).

The 64-cell stage marks the start of a series of invagination-evagination cycles that change the shape of the embryo from spherical to a flattened "prawn chip" (characterized by having a concave side and convex side) and then back to spherical again until gastrulation. Cell divisions occur when the embryo is at its maximum flatness. This pulsing pattern continues for four to five cycles, until the onset of gastrulation ( 18-22 hrs post-fertilization) (Fritzenwanker et al. 2007).

Prior to gastrulation, endodermal fates are specified by canonical Wnt/ $\beta$-catenin and MAPK signaling around the animal pole forming the presumptive endoderm (Wikramanayake et al. 2003; Lee et al. 2007; Röttinger et al. 2012). Gastrulation initiates with formation of a blastopore at the animal pole as the pre-endodermal plate invaginates into the blastocoel, and the blastopore ultimately becomes the oral opening (Fritzenwanker et al. 2007; Lee et al. 2007; Magie et al. 2007). Cellular movements during gastrulation are controlled by a conserved Wnt/PCP/Stbm signaling cascade at the animal pole (Kumburegama et al. 2011) and are typified by apical constriction and weakening of cell junctions followed by invagination of the plate (Figure 7.5d) (Kraus and Technau 2006; Magie et al. 2007). Gastrulation 


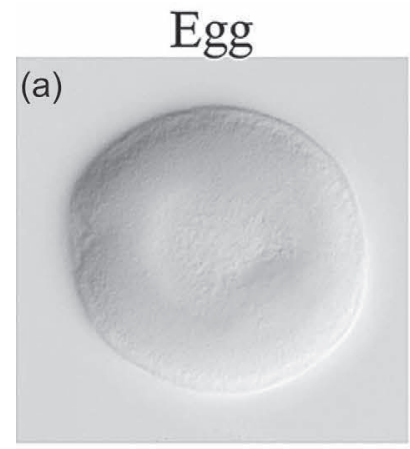

Planula

(e)

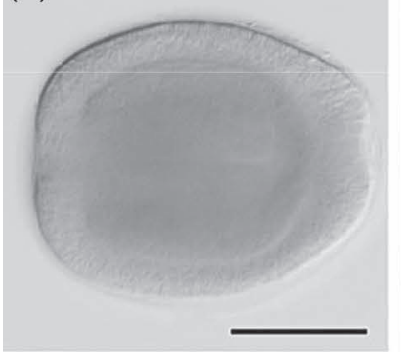

Cleavage

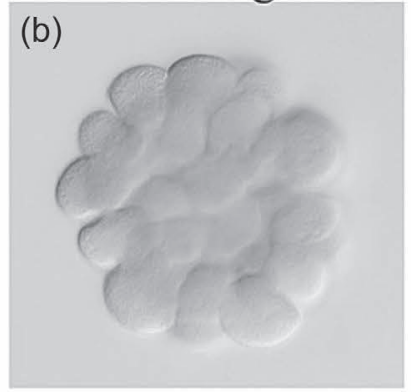

Tentacle Bud

(f)
Blastula

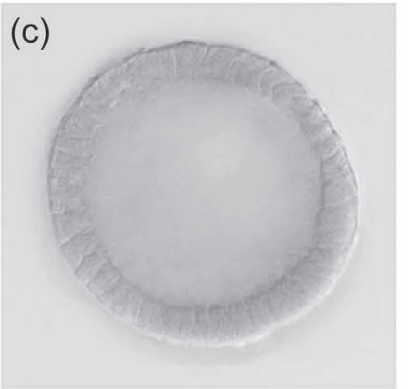

Gastrula

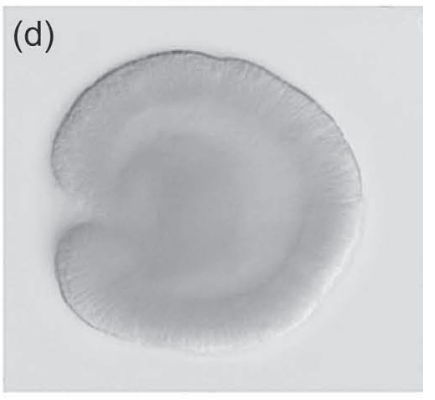

Juvenile Polyp

(g)

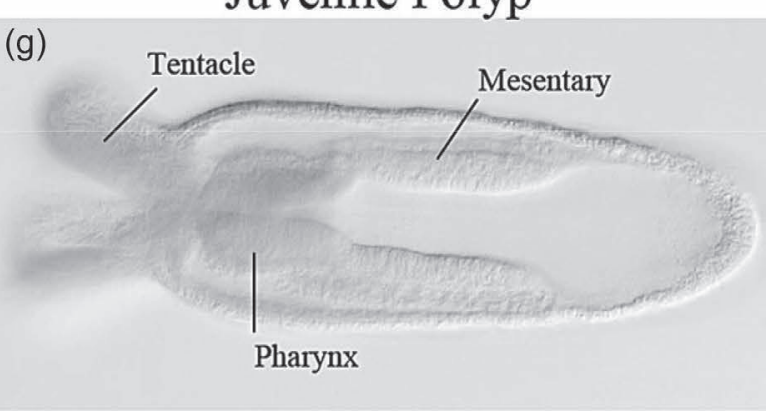

FIGURE 7.5 Developmental stages of Nematostella. Oral end is to the left. Scale bar $=100 \mu \mathrm{m}$.

completes when the ectoderm of the blastopore lip also rolls inward. This ectoderm retains its epithelial organization and gives rise to the pharynx and septal filaments at the tips of the mesenteries.

Following gastrulation, FGF activity at the aboral pole regulates formation of the apical tuft and apical organ (Rentzsch et al. 2008). The resulting planula larvae break out of the egg jelly by $\sim$ two days post-fertilization and are now free-swimming larvae (Figure 7.5e). Planula larvae initially swim in circles, but by $\sim$ three days post-fertilization they exhibit directional swimming with the apical ciliary tuft facing forward (Hand and Uhlinger 1992). The planula stage lasts three to four days, during which the planulae elongate and form the pharynx and the first two (primary) mesenteries (Figure 7.5f) (Fritzenwanker et al. 2007). A heterogeneous distribution of ectodermally derived secretory gland cells is found in the pharynx and mesenteries of the primary polyp, and gene expression studies suggest that development of these cells begins in the planula stage, as the tissues they reside in are formed (Frank and Bleakney 1976; Babonis et al. 2019). Presumptive muscle cells are detected in the early planula with F-actin staining, which becomes concentrated and oriented along the oral-aboral axis in the late planula (Jahnel et al. 2014). Besides the tentacle ring muscles, which are derived from the ectoderm, all other muscle groups are of endodermal origin, with many orthologs of genes that drive muscle development in bilaterians observed in Nematostella during the planula and juvenile polyp stages (Jahnel et al. 2014; Steinmetz et al. 2017). $N v M y H C l$ is first detected at the mid-planula stage and is expressed in retractor muscle cells of both the tentacles and the eight mesenteries of the developing primary polyp (Renfer et al. 2010; Jahnel et al. 2014). The $\mathrm{NvMyHCl}$ transgene is further detected in retractor muscles of adult mesenteries, suggesting muscle cell differentiation in the mesenteries continues in the adult (Renfer et al. 2010).

The four tentacle buds emerge toward the end of the planula stage ( five days post-fertilization) (Figure 7.5f,e). Tentacle primordia are first identified by Fgfrb-positive cells in ring muscle around the oral opening. Stereotyped development and outgrowth of the tentacles is nutrient dependent and driven by crosstalk between TOR-mediated and FGFR signaling pathways. The tentacle buds elongate into the initial tentacles of the juvenile polyp, and Nematostella continue to add tentacles in the adult polyp (Stephenson 1935; Fritz et al. 2013).

Nematostella fully metamorph into juvenile polyps by $6-7$ days post-fertilization (Figure $7.5 \mathrm{~h}$ ). The planula larvae settle with the aboral pole down (Rentzsch et al. 2008), then transform into a tube-shaped polyp with four tentacles around a single oral opening. Growth and maturation of the juvenile polyp into an adult is nutrient dependent, and sexual maturity can be reached in the lab in 10 weeks with regular care and feeding (Hand and Uhlinger 1992).

Neurogenesis begins with the emergence of $N v \operatorname{SoxB}(2)$ and NvAth-like expressing neural progenitor cells in the blastula (Richards and Rentzsch 2014, 2015) and continues throughout development. Molecular regulation of neurogenesis in Nematostella resembles the neurogenic cascades found in bilaterian species (Rentzsch et al. 2017), involving MEK/MAPK (Layden et al. 2016), Wnt (Marlow et 
al. 2013; Sinigaglia et al. 2015; Leclère et al. 2016), BMP (Watanabe et al. 2014; Saina et al. 2009) and Notch (Layden and Martindale 2014; Richards and Rentzsch 2015). Nematostella has both ectodermal and endodermal nerve nets (Nakanishi et al. 2012). Some neural subtypes arise at the same time as their namesake structures (e.g. tentacular neurons in the tentacles and pharyngeal neurons in the pharynx) (Havrilak et al. 2017). Cnidocyte stinging cells are also thought to be neuronal, which is supported by the fact that they require $N v \operatorname{Sox} B(2)$ and $N v \operatorname{Pax} A$ (Babonis and Martindale 2017). Cnidocyte-specific genes and proteins are detected throughout the ectoderm in the early gastrula stage to the primary polyp and in the tentacles and mesenteries of the adult (Zenkert et al. 2011; Babonis and Martindale 2014; Babonis and Martindale 2017).

\subsubsection{Axial Patterning Programs}

Throughout Nematostella development, conserved morphogen gradients and signaling cascades pattern the oral-aboral axis. Wnt/ $\beta$-catenin signaling is a main driver in establishing and patterning the primary oral-aboral body axis and has a role in gastrulation, and high Wnt/Bcatenin promotes oral identity (Wikramanayake et al. 2003; Kusserow et al. 2005; Kraus and Technau 2006; Lee et al. 2007; Marlow et al. 2013; Röttinger et al. 2012; Kraus et al. 2016). $N v$ six $3 / 6$ regulates the aboral domain, and its initial expression is dependent on low Wnt/ $\beta$-catenin in the aboral region (Leclère et al. 2016). Further, a conserved mechanism whereby $\beta$-catenin target genes act to repress aboral gene expression in the oral region represents an ancient regulatory "logic" that may have been present in the urbilaterian ancestor (Bagaeva et al. 2020). The interaction of Wnt/ $\beta$-catenin with specific hox genes further fine-tunes patterning along the oral-aboral axis of the Nematostella embryo and reflects mechanisms of patterning in bilaterians (DuBuc et al. 2018). However, much more work is needed to resolve the role that hox genes have in patterning the primary axis in Nematostella, and whether hox expression can be used to elucidate how the oral-aboral axis relates to the anterior-posterior axis remains a major question (Layden et al. 2016; DuBuc et al. 2018).

The secondary directive axis is established and patterned by graded BMP signaling. Following an initial radial expression in the gastrula around the blastopore, $N v B m p 2 / 4$, $N v B m p 5 / 8$ and $N v C h o r d i n$ become co-expressed on one side (Matus et al. 2006; Rentzsch et al. 2006). Active pSMAD (BMP signal transducer) is concentrated on the opposite side, suggesting a low BMP signal defines the domain and initiates transcription (Saina et al. 2009; Leclère and Rentzsch 2014). Hox genes also play a role in patterning the directive axis in Nematostella. Hox genes control boundary formation, which leads to the radial segmentation of the developing endoderm and positions the eight radial segments along the directive axis-thereby providing their spatial identity (He et al. 2018). Further, cross-regulatory interactions between hox genes occur in both bilaterians and Nematostella during axial patterning (Matus et al. 2006; DuBuc et al. 2018). While many of the same players are involved in patterning the secondary directive and dorsal-ventral axes in Nematostella and bilaterians, respectively, their positions and functions vary (see "Challenging Questions").

\subsubsection{Regeneration}

Many aspects of the regenerative process have been characterized at the behavioral, morphological, cellular and molecular levels (see DuBuc et al. 2014; Bossert and Thomsen 2017). The stages of oral regeneration follow a stereotypic pattern, with initial wound healing complete in $\sim$ six hours post-amputation, and complete regeneration in $\sim$ six to seven days (Figure 7.4c). In subsequent days, the mesenteries fuse, contact the wounded epithelial and then reform the pharynx as new tentacle buds elongate (Amiel et al. 2015). It is hypothesized that a population of quiescent/slow cycling stem cells in the mesenteries are necessary for regeneration (Amiel et al. 2019). Regeneration following bisection occurs at the same rate in both juvenile and adult polyps, is temperature dependent and requires both cellular proliferation and apoptosis (see DuBuc et al. 2014; Bossert and Thomsen 2017). Like what has been observed in other animals that undergo whole-body axis regeneration, some tissue remodeling may also occur during regeneration of oral structures in Nematostella (Amiel et al. 2015; Havrilak et al. 2021). While many of the signaling pathways necessary for Nematostella are redeployed during regeneration, the regulatory logic and the number of genes utilized varies, with unique gene regulatory networks utilized (Warner et al. 2019).

\subsection{GENOMIC DATA}

The generation of the Nematostella genome was a catalyst that greatly advanced the species as a model system and led to a rapid explosion of molecular techniques and publications (Figure 7.2; Table 7.1). The genome was first sequenced and assembled by the Joint Genome Institute in 2007 using a random shotgun strategy and published as a searchable database (https://mycocosm.jgi.doe.gov/Nemve) (Putnam et al. 2007). While this first genome has only partial sequence coverage and is not mapped back to chromosomes, the scaffold organization still informs researchers about syntenic relationships, gene structure and sequence. Improvements to the genome have recently been made with the publication of a second genome (Zimmermann et al. 2020). This new assembly has enhanced sequence coverage and increased chromosomal resolution (https://simrbase.stowers.org).

It was expected that the Nematostella genome would be relatively simple and lack many of the major gene families found in bilaterians. However, bioinformatic analysis uncovered a complex genome comparable in many ways to other animals. It turns out that the Nematostella genome is more like vertebrates than some popular bilaterian models such as Caenorhabditis elegans and Drosophila 
melanogaster (Putnam et al. 2007). The exon-intron structure of Nematostella is like vertebrates and other anemones, which suggests that the eumetazoan ancestor had a similar genetic organization (Putnam et al. 2007). Further, the genome includes major gene families such as wnt (Kusserow et al. 2005), sox (Magie et al. 2005), forkhead (Magie et al. 2005), hedgehog (Matus et al. 2008) and hox (Ryan et al. 2006). Nematostella utilize many major signaling pathways and possess orthologues of many effector genes and antagonists involved in signaling, revealing that the genetic components required for complete signal transduction were established in the cnidarian-bilaterian ancestor (Magie et al. 2005; Putnam et al. 2007; Galliot et al. 2009; Watanabe et al. 2009; Chapman et al. 2010).

The genome has made sequence information easy to access, analyze and manipulate, and allows for the utilization of tools for both discovery-based and comparative genomic studies of varying scales. Sophisticated gene editing is possible using TALEN and CRISPR/Cas9 systems, which can be used to induce targeted mutations and homologous-based recombination, including the generation of transgenic lines and knockout of developmental genes (Ikmi et al. 2014; Servetnick et al. 2017; He et al. 2018). Transcriptomic strategies such as ChIP-seq, RNA-seq and single-cell RNA-seq are now common practice. ChIP-seq studies have led to genome-wide predictions regarding the locations of histone modifications and have demonstrated that there is likely conservation of gene regulatory elements (such as enhancers and promoters) between Nematostella and bilaterians (Schwaiger et al. 2014; Technau and Schwaiger 2015; Rentzsch and Technau 2016). ChIP-seq experiments suggest acetylated histones are enriched in the 5' proximal region of gene promoters (and sometimes in the first intron) of genes they control, which facilitates identification of regulatory elements used to generate transgenic reporters. Transgenic animals have been successfully generated by capturing and cloning $\sim 1.5-2.5 \mathrm{~KB}$ of the region upstream of the transcription start site (Renfer et al. 2010; Nakanishi et al. 2012; Layden et al. 2016; Renfer and Technau 2017). RNA-seq and microarrays have been used to profile gene expression levels during development and regeneration (Tulin et al. 2013; Helm et al. 2013; Fischer et al. 2014; Warner et al. 2018). The compilation of these RNA-seq studies into Nvertx, a searchable database, allows for quick comparison between timepoints and/or between the processes of development and regeneration (http://nvertx.ircan.org) (Warner et al. 2018, 2019). These databases are powerful tools because a researcher can evaluate their findings relative to this published source or can check expression profiles and make and test initial hypotheses about potential candidate genes before doing any functional studies themselves. Single-cell RNA-seq studies are now possible, and initial studies have used similarities in cellular expression profiles to generate testable hypotheses regarding cell types, their diversity and their functions (http://compgenomics.weizmann.ac.il/ tanay/?page_id=724) (Sebé-Pedrós et al. 2018).

\subsection{METHODS AND FUNCTIONAL APPROACHES}

\subsubsection{Culture and Care}

Establishing and maintaining a lab population of Nematostella is simple and economical. Founder animals can be purchased from commercial vendors, requested from other laboratories or collected from the field using minimal equipment (see Stefanik et al. 2013). Animals can be kept in glass bowls in a cool dark room, and their husbandry only requires regular brine shrimp feeding and weekly water changes (Stephenson 1935; Williams 1983; Hand and Uhlinger 1992). They can also be maintained in modified fish aquaculture systems for large-scale cultures. Population size can be increased through sexual reproduction, and clonal lines can be developed by allowing animals to asexually reproduce or by cutting adults to create regenerates (Figure 7.4) (Hand and Uhlinger 1992; Reitzel et al. 2007; Stefanik et al. 2013). Nematostella spawn year-round in culture (Hand and Uhlinger 1992; Fritzenwanker and Technau 2002). Under laboratory conditions, spawning is induced by exposing animals to a light source and by increasing temperature (Niehrs 2010; Genikhovich and Technau 2017).

\subsubsection{Behavioral and Ecological Approaches}

The fact that these animals are found in abundance in shallow estuarine environments makes them easy to find, collect and manipulate for field studies. Due to their mostly sedentary and infaunal nature, controlled field experiments can be easily conducted without the worries of tracking individuals or animals escaping from experimental areas. Water-permeable cages allow for testing under natural conditions and provide a way to control the contents of the cage, including what can enter and exit it. For example, cages placed within natural habitats have been used to track changes within a population under different conditions, including those in which predators, food availability and abiotic environmental factors were varied (Wiltse et al. 1984; Tarrant et al. 2019). Nematostella can tolerate a wide range of environmental parameters and are often found living at the extremes of their tolerable ranges for temperature, salinity and oxidative stress (Williams 1983; Hand and Uhlinger 1992; Reitzel et al. 2013; Friedman et al. 2018). This remarkable environmental phenotypic plasticity makes them an intriguing indicator species and a potential model for studies of stress tolerance, effects of the environment on development, community structure and adaptive evolution. Further, existing information regarding population genetic structure, gene flow and protein-coding polymorphisms allows for studies to be placed in a broader evolutionary context (Darling et al. 2004; Reitzel et al. 2013; Friedman et al. 2018). The broad molecular toolbox available for Nematostella allows field researchers to take an integrative approach to experiments (Table 7.1). 
Nematostella is amenable to both field and lab studies. Because it is an established laboratory model with a published genome, it is possible to determine the mechanisms of molecular, cellular and behavioral changes that occur in the wild due to environmental changes or following manipulations in a laboratory environment. Several naturally occurring behaviors have been described in Nematostella, including burrowing, creeping, climbing, feeding, contracting, spawning, fissioning and the propagation of peristaltic waves (Hand and Uhlinger 1992, Hand and Uhlinger 1995; Williams 2003; Faltine-Gonzalez and Layden 2019; Havrilak et al. 2020). Despite it often being difficult to observe behaviors in the field due to their small size, infaunal nature and usually low water clarity, behavioral observations can be done in the lab where video recording and magnification are easily accomplished and natural conditions can be mimicked. Besides studying behavioral observations to understand the behavioral ecology of Nematostella, behaviors can be used as an experimental readout due to the depth at which many behaviors have been described (e.g. Williams 2003). For instance, one can assess behaviors as a means of determining the effect of a treatment (e.g. following drug treatments, genetic manipulations) or as a measure for the completion of morphogenesis (e.g. during growth/degrowth, regeneration) (Figure 7.6) (Faltine-Gonzalez and Layden 2019; Havrilak et al. 2021).

\subsubsection{Tissue Manipulation and Tracking}

Classical embryological techniques, such as embryo separation, dye tracing during embryo development and tissue grafting (Lee et al. 2007; Nakanishi et al. 2012; Steinmetz et al. 2017; Warner et al. 2019), are feasible due to large transparent embryos and adults. Dissection and transplantation of fluorescent tissue from transgenic embryos into developing wild type embryos have allowed researchers to begin constructing a fate map of the germ layers, and these techniques could be useful in further constructing the Nematostella fate map (Steinmetz et al. 2017). Researchers have successfully cultured sheets of ectodermal tissue, which was able to transform into 3D structures and be sustained for several months (Rabinowitz et al. 2016). Cell culture techniques are being developed in Nematostella and are expected to be possible due to the success of tissue culture and recent

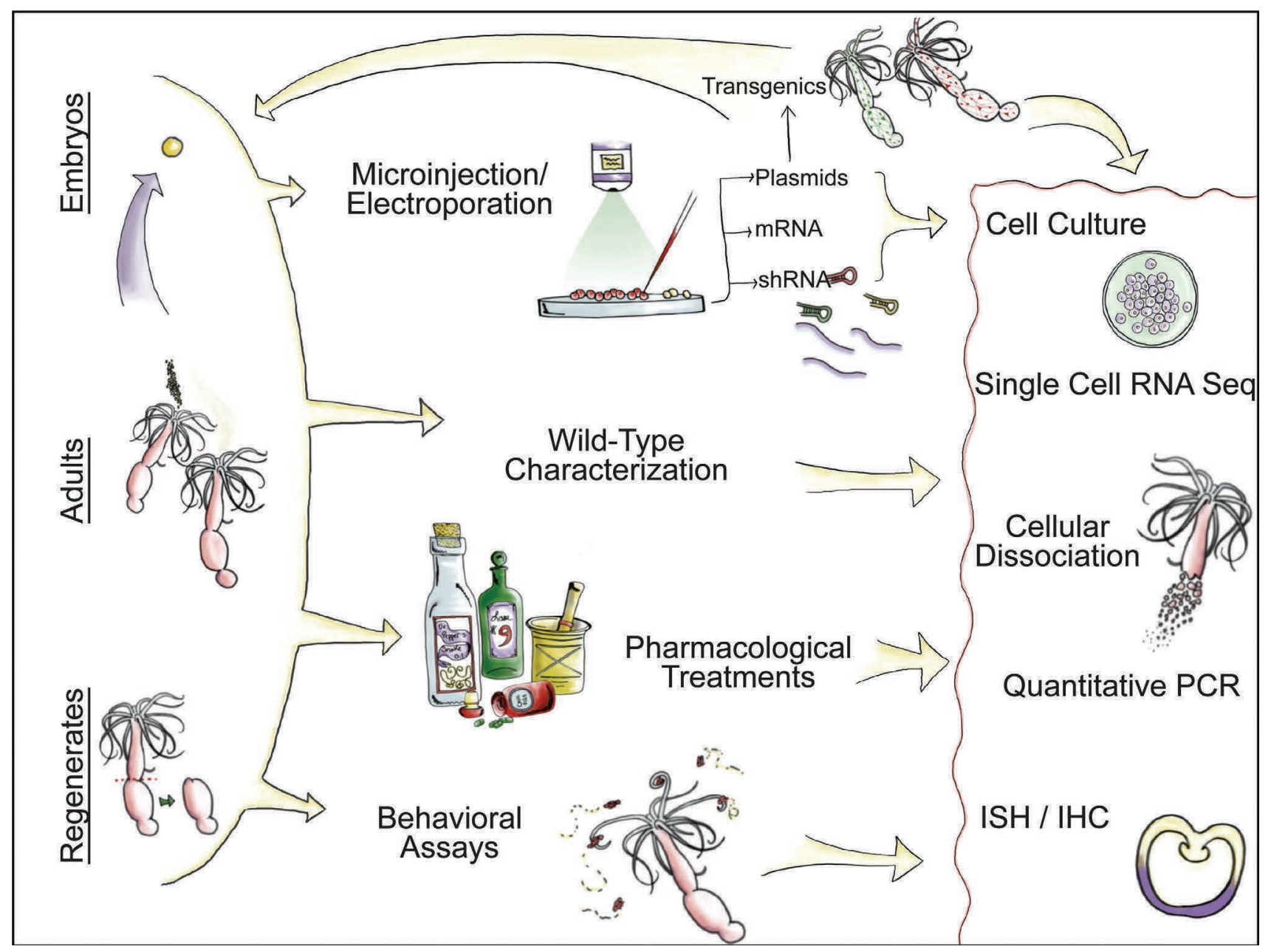

FIGURE 7.6 Potential workflow showing integration of multiple techniques using Nematostella. 
ability to successfully dissociate animals into their cellular components (see the following paragraph). These culturing methods would allow research to be focused on a specific tissue or cell type and could negate the need to maintain an animal population due to the ability to freeze cell stocks (e.g. Fricano et al. 2020).

Dissociation of cells from transgenic and wild type adult animals has been accomplished using different combinations of enzymatic, chemical and mechanical techniques (Sebé-Pedrós et al. 2018; Clarke et al. 2019; Torres-Méndez et al. 2019; Weir et al. 2020). Cellular dissociation has also allowed for studies of cellular adhesion using the hanging drop method to study reaggregation (Clarke et al. 2019). Further, cellular dissociation has opened the door to the field of electrophysiology. For example, single-cell recordings from nematocytes have given us insight into the physiology of a novel cell type and bettered our understanding of how Nematostella distinguish salient environmental information to regulate cnidocyte firing (Weir et al. 2020), contributing to our understanding of cnidarian sensory systems and their stinging response.

\subsubsection{Detection of Cellular Processes}

The relatively simple body plan of Nematostella, consisting of only two transparent tissue layers, facilitates the use of common labeling techniques to investigate cellular processes utilized during morphogenesis and homeostasis. Standard techniques for cellular proliferation have been used by labeling animals with EdU and BrdU (Passamaneck and Martindale 2012; Richards and Rentzsch 2014; Amiel et al. 2015; Rabinowitz et al. 2016; Warner et al. 2019; Havrilak et al. 2021). TUNEL assays have been used to detect apoptotic cells during development and regeneration (Warner et al. 2019; Zang and Nakanishi 2020).

\subsubsection{Regeneration}

Inducing a regenerative response in Nematostella is simple, and the process has been characterized at many levels. Regeneration is induced by wounding the animal with a scalpel or probe (see DuBuc et al. 2014; Bossert and Thomsen 2017). The wound site and severity of the injury inflicted are dictated by the research question. Typically, studies have focused on whole-body axis regeneration, where live animals are bisected along the body column into oral and aboral halves and regeneration of one or both fragments is observed (Figure 7.4c) (Passamaneck and Martindale 2012; Amiel et al. 2015; Schaffer et al. 2016). However, a more acute regenerative response can be triggered following a focal injury where whole-body axis regeneration is not required (e.g. tentacle amputation, puncture wound, incomplete bisection along the body column) (Reitzel et al. 2007; DuBuc et al. 2014). This flexibility in the regeneration paradigm allows for a variety of questions to be asked. The ability to document gene expression in different regenerative paradigms, as well as to compare it to development, will continue to make this a fruitful area of research in this model. For example, many hypotheses can be tested due to comparative transcriptome analysis using RNAseq during the regeneration of oral vs. aboral fragments-which identified similarities and differences in gene expression profiles between the two halves (Schaffer et al. 2016). Methods for assessing wound closure, and detailed descriptions of key morphological landmarks that occur throughout the process of regeneration, have been described and can be used to assess the progress of the regenerative response (Bossert et al. 2013; Amiel et al. 2015). Assaying the regenerative phenotype following pharmacological or genetic manipulation could be used to understand the mechanisms of regeneration (e.g. using an inducible promoter or knockout transgenic line). Transgenic reporter lines allow for the tracking of specific cell types in live animals, including specific neural subtypes, which has made the regeneration of the nerve net tractable (Figure 7.6) (Layden et al. 2016; Havrilak et al. 2017; Sunagar et al. 2018; Havrilak et al. 2021).

\subsubsection{Genetic Approaches}

\subsubsection{Microinjection and Electroporation}

Molecules can be introduced into live embryos using microinjection and electroporation techniques, which facilitate the delivery of compounds such as shRNA, mRNA, morpholinos and plasmids into eggs. With microinjection, a very fine glass needle is used to penetrate an egg and deliver a small volume of the loaded injection mixture using forced air (Layden et al. 2013; Renfer and Technau 2017; Havrilak and Layden 2019). An experienced researcher can inject thousands of embryos in a single session. Microinjection offers more experimental utility due to the variety of molecular compounds that can be injected, ranging from plasmids to shRNAs. Microinjection has been used successfully for genetic knockdown and misexpression experiments, as well as for the generation of transgenic animals in Nematostella (Layden et al. 2013; Ikmi et al. 2014; Renfer and Technau 2017). Electroporation offers a simple and quick method for the delivery of molecules into hundreds of animals simultaneously by generating electrical pulses that create pores in the plasma membrane that allow small molecules to be taken up. So far, this method has only proved successful in the delivery of shRNA for knockdown experiments in Nematostella (Karabulut et al. 2019).

\subsubsection{Gene Disruption}

Tools for both gain and loss of function experiments are available (Table 7.1). Injection of in vitro synthesized mRNA allows for a gene of interest to be overexpressed (Wikramanayake et al. 2003), while introduction of shRNA or morpholinos facilitates genetic knockdown of a gene of interest (Magie et al. 2005; Rentzsch et al. 2008; He et al. 2018). Gene editing technologies can also be used to silence, move, knock down or overexpress a particular gene in both F0 and F1 generation mutants, and pharmacological treatments can also 
be performed for gain and loss of function experiments (see "Transgenics and Pharmacological Manipulations").

\subsubsection{Transgenics}

Generation of transgenic animals utilizing tissue and cell typespecific promoters driving a fluorescent tag and/or specific gene of interest has been successful using random meganuclease-assisted integration (Figure 7.3b) and site-specific CRISPR/Cas9 homologous recombination (Ikmi et al. 2014; Renfer and Technau 2017). Promoter sequences have been captured by cloning $1.5-2.5 \mathrm{~kb}$ of the genetic sequence upstream from the coding sequence of a gene of interest (Putnam et al. 2007; Renfer et al. 2010; Nakanishi et al. 2012; Layden et al. 2016; Renfer and Technau 2017). Transgenic lines have been made with broad expression using promoter sequences such as actin, ubiquitin and elongation factor 1a (Fritz et al. 2013; Steinmetz et al. 2017; He et al. 2018), and promoters for tissue and cell specific genes have also been utilized for restricted expression such as myosin heavy chain and $\operatorname{sox} B(2)$ (Renfer et al. 2010; Richards and Rentzsch 2014). A plasmid backbone containing I-sceI meganuclease recognition sites is available (AddGene.org: plasmid \#67943) and allows for the desired construct to be swapped out using basic cloning strategies (Renfer et al. 2010). Gene editing has been achieved through homologous recombination using TALEN and more frequently CRISPR/Cas9 (Ikmi et al. 2014; Zang and Nakanishi 2020). For CRISPR/Cas9, a plasmid containing homology arms for a Nematostella-specific $F p 7$ locus allows for expression or disruption of a desired gene of interest. Importantly, the $F p 7$ locus can be disrupted without detrimental effects on the animal and allows for easy screening due to the loss of endogenous red fluorescent protein following cassette insertion. The application of conditional promoters, including an already identified heat shock promoter, opens the door for temporal control of gene expression and disruption in the future (Ikmi et al. 2014).

\subsubsection{Visualizing Gene Expression}

Several tools are available in Nematostella for visualizing spatial and temporal differences in gene expression. Both colorimetric and fluorescent whole mount in situ hybridization are widely used for determining spatial expression of mRNA at specific time points during development and regeneration (Niehrs 2010; Genikhovich and Technau 2017). Immunohistochemistry has been used to visualize protein expression (Zenkert et al. 2011; Wolenski et al. 2011;

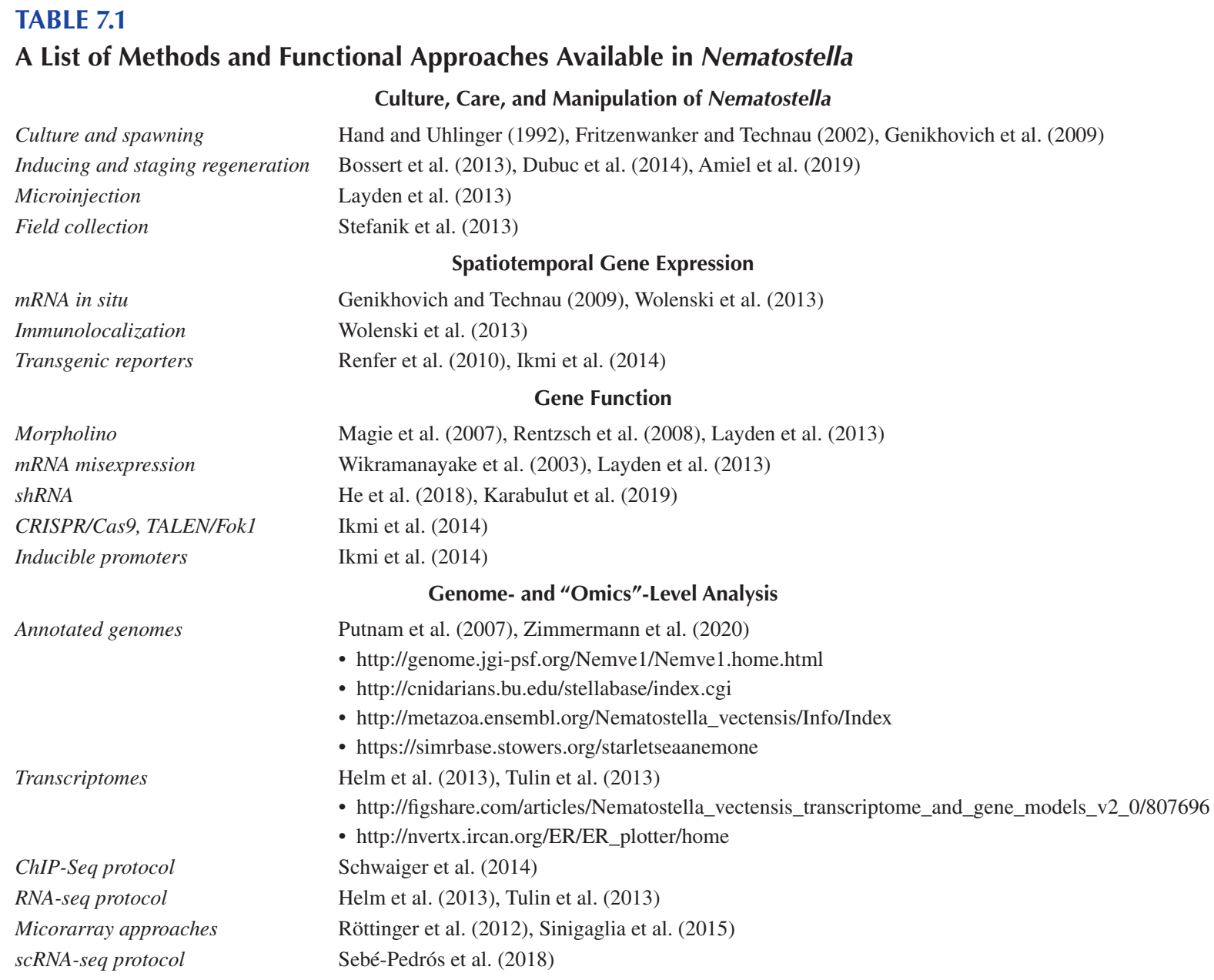


Nakanishi et al. 2012; Zang and Nakanishi 2020). Transgenic reporter lines provide another means of assaying spatial and temporal protein expression and allow for live visualization and imaging. Quantitative real-time polymerase chain reaction is a quick method for determining mRNA expression levels at a given point in time and is often used experimentally in tandem with in situ hybridization and in the confirmation of sequencing results. Together these methods are powerful tools for characterizing wild type and transgenic expression and as a readout for gain and loss of function experiments (Figure 7.6).

\subsubsection{Genome- and "Omics"-Level Approaches}

Genomic- and transcriptomic-level experiments under varying developmental, regenerative and/or environmental paradigms are possible since the publication of the genome. ChIP-seq studies can be used to determine epigenetic protein interactions with open chromatin. For example, ChIPseq can uncover potential genomic interactions of a protein of interest or facilitate the identification of regulatory elements for a gene of interest (Schwaiger et al. 2014; Technau and Schwaiger 2015; Rentzsch and Technau 2016). RNA-seq and single cell RNA-seq have allowed for the investigation of global gene expression levels in whole animals and single cells, respectively (Tulin et al. 2013; Helm et al. 2013; Fischer et al. 2014; Warner et al. 2018; Sebé-Pedrós et al. 2018). Ultimately, each of these methods provides different levels of resolution, and the method used will depend on the question being asked.

\subsubsection{Pharmacological Manipulation}

Pharmacological agents have been used to target specific developmental pathways, as well as to target pathways to alter the physiology of the adult animal. Administering pharmacological agents requires only introducing the desired concentration to the sea water in which treatment animals are growing. Treatments can be administered at any stage from developing embryos to mature adults. Pharmacological agents offer a quick and easy way to target pathways in a high-throughput manner. It is possible to alter basic cellular processes using drug treatments. For example, cell proliferation has been blocked with hydroxyurea (Amiel et al. 2015). Wnt/B-catenin activity can be overactivated using 1-azakenpaullone and/or alsterpaullone, and inhibited using iCRT14 (Trevino et al. 2011; Watanabe et al. 2014). The gamma secretase inhibitor DAPT can be given to effectively disrupt the Notch/Delta pathway (Layden and Martindale 2014), and the receptor tyrosine kinase inhibitor SU5402 can be used to effectively inhibit Fgf receptors (Rentzsch et al. 2008). Additionally, the mTOR pathway can be disrupted by bathing animals in rapamycin (Ikmi et al. 2020). While many of the treatments discussed previously would typically be applicable to developing animals, there are also several agents that can alter the physiology of adult Nematostella. For example, bathing adults in the neurotransmitter acetylcholine can induce tentacle contractions, while lidocaine can suppress these contractions (Faltine-Gonzalez and Layden 2019).

\subsubsection{InTEGRATION OF ApProaches}

While the approaches discussed here are organized into subsections, there is no hard line defining what they can be used for. The combination of various tools from field approaches to molecular, cellular and behavioral techniques can be combined to address a nearly limitless range of questions (Figure 7.6). Following the establishment of a lab population, a basic molecular biology lab setup will allow a researcher to tackle questions pertaining to the fields of molecular ecology, mechanisms of behavior, evolution, development, regeneration and so on (Figure 7.6). There is also the expectation that the Nematostella model will keep up with major advances in technology, since cutting-edge techniques continue to become available in this system. Advances in single-cell technologies and the application of conditional/inducible alleles will further refine the resolution and control at which experiments can be performed. Adding in the fact that field and lab comparisons and/or wild type and transgenic comparisons can be included as additional variables makes it so researchers have a high level of control, allowing them to implement experimental parameters beyond those offered by other model systems.

\subsection{CHALLENGING QUESTIONS}

\subsubsection{Is There a Deep Evolutionary Origin for Key Bilaterian Traits?}

An explosive radiation of taxa occurred within the bilaterian lineage, and it is believed to be due to the evolution of several unique characteristics (e.g. mesodermal germ layer and bilateral symmetry) that allowed them to occupy previously inaccessible niches. The evolution of these traits allowed for the evolution of larger, more complex body plans and increased specialization of structure organization and function-including cephalization and the centralization of nervous systems. Understanding the mechanisms that led to the bilaterian radiation is a longstanding evolutionary question that can only be answered by studying animals that are closely related to bilaterians in order to infer what molecular tool-kit was available to their common ancestor. Cnidarians are regarded as the sister taxon to the bilaterians (Wainright et al. 1993; Medina et al. 2001; Collins 2002), and therefore cnidarian models, such as Nematostella, offer an appropriate outgroup species to study the molecular basis for the origin of key bilaterian traits, such as the mesoderm and bilaterality, because they allow us to deduce the evolutionary history of these derived traits (Figure 7.7). In fact, Nematostella first gained momentum as a model species for its utility in uncovering the evolutionary mechanisms that led to key bilaterian features.

\subsubsection{Origin of the Mesoderm}

Thus far, studies with Nematostella have used comparative genetic approaches and germ layer fate mapping to 
form different hypotheses regarding the molecular basis of mesoderm evolution (Scholz and Technau 2003; Martindale et al. 2004; Steinmetz et al. 2017; Wijesena et al. 2017). One hypothesis suggests that the mesoderm was derived from a dual-functional endoderm originating in the diploblastic ancestor, termed the "endomesoderm", which performs both traditional endodermal and mesodermal functions within a single germ layer (Martindale et al. 2004; Wijesena et al. 2017). Expression of genes restricted to the mesoderm in bilaterians were found in the endoderm of Nematostella, leading to the "endomesoderm" hypothesis (Martindale et al. 2004). Further, expression of a conserved set of genes involved in the gene regulatory network driving heart field specification in bilaterian mesoderm was found to be functional in the endoderm of Nematostella at early developmental stages (Wijesena et al. 2017). Since they lack a closed circulatory system and other mesenchymal cell types, this begs the question: What are the functions of these heart field and other traditionally mesoderm-specific genes in Nematostella?

Other studies have tested the "endomesoderm" hypothesis and arrived at a different model of mesoderm evolution. Germ layer fate mapping showed that the pharynx and mesenteries are composed of cells derived from both germ layers, as opposed to being derived from only the endoderm as previously thought, and gene expression experiments suggested that these structures are also functionally partitioned. Further, the Nematostella endoderm has an expression profile that resembles bilaterian mesoderm (e.g. heart and gonadal genes), and the pharyngeal ectoderm expresses genes common to bilaterian endoderm (e.g. gut-specific digestion genes) (Steinmetz et al. 2017). These data point to an alternate model of germ layer homology where the cnidarian pharyngeal ectoderm is analogous to the bilaterian endoderm, and the cnidarian endoderm is analogous to the bilaterian mesoderm, supporting a proposed mechanism for bilaterian mesoderm formation where the expansion of the pharyngeal ectoderm down into the body cavity led to the formation of an internal mesodermal layer in a prebilaterian ancestor (Steinmetz et al. 2017; Steinmetz 2019). Support for this model requires functional studies to show that the gene expression profiles of Nematostella not only correspond to bilaterian germ layer profiles but also have homologous functions.

Both hypotheses propose that the cnidarian endoderm has analogous function to the bilaterian mesoderm. The main difference lies in whether the pharynx and mesenteries contain both ectodermal and endodermal tissues and function as bilaterian endoderm and mesoderm, respectively. To reconcile these different hypotheses, better resolution of gene regulatory networks in adult animals is needed in order to ascertain if mesodermal gene expression and function (such as the heart field specification network) are restricted to the endodermal portions of the bi-layered mesenteries and pharynx.

\subsubsection{Mechanisms of Axial Patterning Leading to Bilaterality}

Despite the seemingly endless variation in animal body plans, all taxa appear to have clear regimented developmental programs that set up the body axes that give rise to the

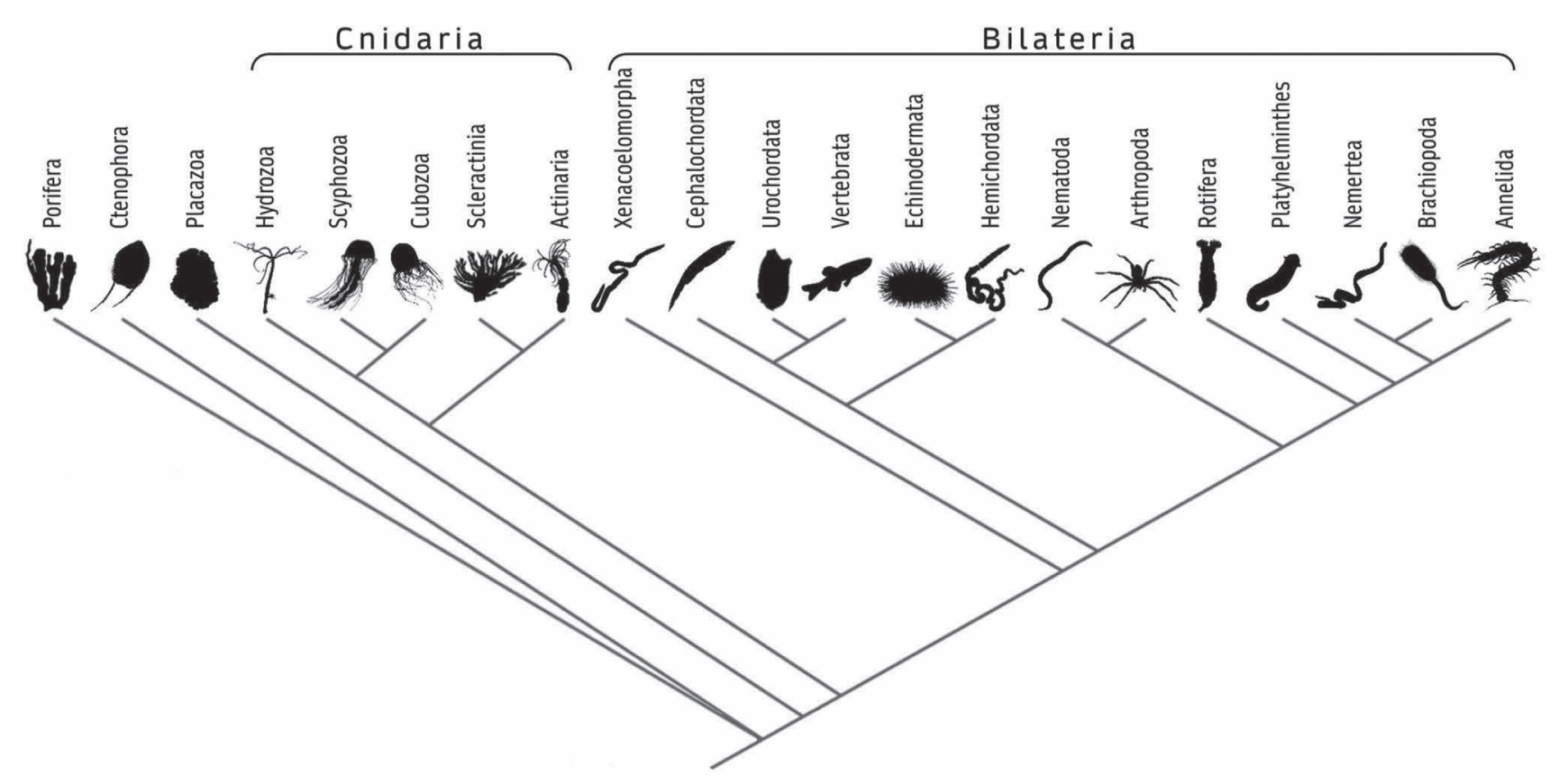

FIGURE 7.7 Phylogeny showing relationships between cnidaria, bilateria and early metazoa. Nematostella is an actinarian cnidarian. Cnidarians and bilaterians are sister taxa. Porifera and placazoa lineages are shown sharing a node because their phylogenetic position is unresolved. 
unique morphology of each species. Many similarities in the mechanics of axial patterning have been observed between taxa. This has led to the question: Is there a conserved molecular program central to axial patterning that has been co-opted across evolutionary time?

At first glance, Nematostella appear radially symmetrical, with their body plan centered around a primary oral-aboral axis that runs the length of the body column. However, upon closer examination of the structural features of Nematostella, it is evident that they possess bilateral symmetry along a secondary "directive" axis that runs perpendicular to the primary axis (see "Anatomy"). At a molecular level, the perpendicular primary and secondary axes are derived from orthogonal morphogen gradients that work in concert to set up the body plan. In bilaterians, the orthogonal arrangement of morphogen gradients is also a fundamental aspect of body axis patterning (Niehrs 2010; Genikhovich and Technau 2017).

Major morphogen signaling pathways, with an established role in bilaterian anterior-posterior axial patterning, play a similar role in setting up domains along the oralaboral axis in Nematostella (Leclère et al. 2016; Amiel et al. 2017; Bagaeva et al. 2020). Like bilaterians, a Wnt/ $\beta$-catenin gradient, with a similar regulatory logic, is established along the primary axis (Marlow et al. 2013, Kraus et al. 2016, Bagaeva et al. 2020). In bilaterians, a key factor in forming the dorsal-ventral axis is the establishment of opposing gradients of bone morphogenic protein and its antagonist chordin on opposite ends of the secondary axis, perpendicular to the primary axis (Niehrs 2010, Genikhovich and Technau 2017). In contrast, expression domains of bone morphogenetic protein and chordin overlap and are on the same side of the directive axis in Nematostella (Matus et al. 2006; Rentzsch et al. 2006; Leclère and Rentzsch 2014). This suggests evolutionary plasticity in the BMP/Chordin systems but does not answer how they functioned and were co-opted in the establishment of diverse secondary axial patterning programs throughout evolutionary history. It is worth noting that besides these two major upstream morphogen pathways, other factors, such as hox genes, play critical roles as downstream effectors in shaping and refining axial patterning in Nematostella and bilaterians (Graham et al. 1991; Pearson et al. 2005; DuBuc et al. 2018; He et al. 2018).

The accumulation of data thus far suggests deep evolutionary roots for the morphogenetic programs governing axis patterning (Matus et al. 2006; Bagaeva et al. 2020), regardless of body plan complexity. Although the same morphogenetic pathways seem to play an important role in patterning the primary and secondary axes in Nematostella, there appear to be key differences in how morphogens are spatially distributed and interacting (Matus et al. 2006; Rentzsch et al. 2006; Leclère and Rentzsch 2014). Similarities in axial programming between Nematostella and bilaterians make them an ideal candidate for understanding if/when a general morphogenetic program was co-opted for the evolution of bilateral symmetry. Comparisons with other cnidarians and early metazoans will help to resolve how these patterning mechanisms evolved and functioned in the urbilaterian ancestor and prior to the cnidarian-bilaterian split.

\subsubsection{Can Nematostella Be Used as a CNidarian Model for Cnidarians?}

Establishing a genetically amenable, high-throughput, cnidarian model would improve our understanding of many aspects of cnidarian biology, which has been hindered by our inability to easily access, observe and culture many species within this phylum. A major question is: Can we better understand the effects of the changing environment and inform conservation strategies by utilizing established cnidarian models that are amenable to high-throughput laboratory techniques? Although corals can be harvested and kept under laboratory conditions (provided that specific environmental parameters are met) their natural history makes it very difficult to control spawning behavior and therefore makes it so that embryos are only available up to a few times a year (Harrison et al. 1984; Baird et al. 2009; Keith et al. 2016; Craggs et al. 2017; Pollock et al. 2017; Cleves et al. 2018). In addition, there are few tools and resources available for conducting molecular, cellular or physiological research in non-model cnidarian systems (Technau and Steele 2011). A notable exception is a study that used CRISPR/Cas9 in the coral Acropora millepora to target a few genes of interest. However, to obtain embryos, prior knowledge of when spawning would occur was necessary so that corals could be harvested and brought into the lab just prior to their natural spawning event (Cleves et al. 2018). This exemplifies the logistical hurdles that are often present in coral and other cnidarian research.

An intriguing possibility is that Nematostella could be employed as a cnidarian model for cnidarians due to the repertoire of tools available and easy culture. Nematostella has no symbionts, and therefore it would not be useful in modeling symbiotic relationships. However, the fact that we can easily manipulate Nematostella at a molecular level sets it up as a good proxy to investigate fundamental molecular programs in other cnidarians. This way, hypotheses could be quickly tested in this developed model so that resources can be mobilized most efficiently in hard to study cnidarian species. An additional question is: Can Nematostella be used as a cnidarian model for environmental stress tolerance and adaptation? This could broaden our understanding of how imperiled cnidarians are likely to cope with ongoing environmental change. Plus, understanding the underlying mechanisms responsible for environmental plasticity in Nematostella could potentially be exploited in the conservation of other species.

\subsubsection{How Do Novel Cell Types Evolve?}

Longstanding evolutionary questions are: How does evolutionary novelty arise, and how does novelty lead to major evolutionary transitions? To investigate these questions 
requires a model that possesses cell types with true morphological and functional novelty. Cnidocytes are phenotypically unique stinging cells and a defining characteristic of the cnidarian phylum. Cnidocytes are one of only a handful of examples of an unequivocal evolutionary novelty and thus offer a unique opportunity to investigate the mechanisms that lead to evolutionary novelty-something that is not possible in many model systems. Using Nematostella, studies can be focused on the molecular basis of cnidocyte development (Babonis and Martindale 2017; Sunagar et al. 2018). This will inform how newly generated genes/proteins interact with existing biological programs, leading to the emergence of novel proteins and, in turn, cell types (Babonis and Martindale 2014; Babonis et al. 2016; Layden et al. 2016).

\subsubsection{Does Regeneration Recapitulate Development?}

Unraveling the molecular basis of development and regeneration is pivotal to answering the question of whether developmental programs are co-opted for regenerative processes. Complicating matters is that historically, researchers were limited by models suited to either the study of development or regeneration or those that had limited regenerative capabilities (e.g. Ambystoma mexicanum, Danio rerio, Xenopus laevis). Models where both processes can be examined within the same species took longer to become established (e.g. Nematostella vectensis, Hofstenia miamia). A distinguishing feature of Nematostella is that it is capable of whole-body axis regeneration. This, coupled with the fact that it is becoming a strong model for development, offers the unique ability to directly compare these two processes within the same animal. Studies in Nematostella and other species are gaining support for the hypothesis that regeneration is only a partial redeployment of embryonic development (e.g. Schaffer et al. 2016; Warner et al. 2019).

Moving forward, it will be necessary to study whether the same program differences arise regardless of regeneration paradigm. For example, during whole-body axis regeneration in Nematostella, the initial regenerative response of certain neural subtypes differs under varying regenerative paradigms, suggesting that there may be cell type differences (Havrilak et al. 2021). As we gain functional understanding of these processes, it begs the question of whether we can unlock regenerative potential in non-regenerative models and use this knowledge to develop medical therapies.

\subsubsection{Other Challenging Questions}

The topics addressed previously are only a small subset of the challenging questions that Nematostella is poised to address. For example, other questions pertaining to evolution and development, such as the centralization of the nervous system within the bilaterian lineage, are possible because of the position of cnidarians as sister taxa. Outside of its use in academia, there is also definite potential for Nematostella within applied research fields, such as biotechnology.
Within the biotech industry, one innovative group looks to use Nematostella to help consumers combat the signs of ageing by harnessing the stinging action of cnidocyte cells to optimize the delivery of skin care agents deep into the skin (Toren and Gurovich 2016). Although few other examples of Nematostella in applied research exist, it is easy to imagine other uses for this cnidocyte-mediated injection technology throughout the beauty and medical industries, as well as many other untapped applications waiting to be uncovered.

\section{ACKNOWLEDGMENTS}

Thanks to Anna Delaney and Mark Williams for uncovering archival information on Miss. Gertrude F. Selwood from when she was at their respective universities. We thank Eric Röttinger for the photograph of the adult Nematostella, MingHe Chen for developmental images and Dylan FaltineGonzalez for insightful discussions.

\section{BIBLIOGRAPHY}

Amiel, A.R., Foucher, K., Ferreira, S., and E. Röttinger. 2019. Synergic coordination of stem cells is required to induce a regenerative response in anthozoan cnidarians. BioRxiv. December 31:891804.

Amiel, A.R., Hereroa J., Nedoncelle, K., Warner, J., Ferreira, S., and E. Röttinger. 2015. Characterization of morphological and cellular events underlying oral regeneration in the sea anemone. Nematostella vectensis. International Journal of Molecular Sciences. 16:28449-28471.

Amiel, A.R., Johnston, H., Chock, T., Dahlin, P., Iglesias, M., Layden, M., Röttinger, E., and M.Q. Martindale. 2017. A bipolar role of the transcription factor erg for cnidarian germ layer formation and apical domain patterning. Developmental Biology. 430:346-361.

Babonis, L.S., and M.Q. Martindale. 2014. Old cell, new trick? Cnidocytes as a model for the evolution of novelty. Integrative and Comparative Biology. 54:714-722.

Babonis, L.S., and M.Q. Martindale. 2017. PaxA, but not PaxC, is required for cnidocyte development in the sea anemone Nematostella vectensis: EvoDevo. 8:14.

Babonis, L.S., Martindale, M.Q., and J.F. Ryan. 2016. Do novel genes drive morphological novelty? An investigation of the nematosomes in the sea anemone Nematostella vectensis. BMC Evolutionary Biology. 16:114.

Babonis, L.S., Ryan, J.F., Enjolras, C., and M.Q. Martindale. 2019. Genomic analysis of the tryptome reveals molecular mechanisms of gland cell evolution. EvoDevo. 10:23.

Bagaeva, T., Aman, A.J., Graf, T., Niedermoser, I., Zimmermann, B., Kraus, Y., Schatka, M., Demilly, A., Technau, U., and G. Genikhovich. 2020. $\beta$-catenin dependent axial patterning in Cnidaria and Bilateria uses similar regulatory logic. BioRxiv. September 8:287821.

Baird, A.H., Guest, J.R., and B.L. Willis. 2009. Systematic and biogeographical patterns in the reproductive biology of Scleractinian corals. Ecology, Evolution, and Systematics. 40:551-571.

Berking, S. 2007. Generation of bilateral symmetry in Anthozoa: A model. Journal of Theoretical Biology. 246:477-490.

Bossert, P.E., Dunn, M.P., and G.H. Thomsen. 2013. A staging system for the regeneration of a polyp from the aboral 
physa of the anthozoan cnidarian Nematostella vectensis. Developmental Dynamics. 242:1320-1331.

Bossert, P.E., and G.H. Thomsen. 2017. Inducing complete polyp regeneration from the aboral physa of the starlet sea anemone Nematostella vectensis. Journal of Visualized Experiments. 119.

Brandão, R.A., Gusmão, L.C., and P.B. Gomes. 2019. Diversity of Edwardsiidae sea anemones (Cnidaria: Anthozoa: Actiniaria) from Brazil, with the description of a new genus and species. Journal of the Marine Biological Association of the United Kingdom. 99:1087-1098.

Chapman, J.A., Kirkness, E.F., Simakov, O., Hampson, S.E., Mitros, T., Weinmaier, T., Rattei, T., Balasubramanian, P.G., Borman, J., Busam, D., Disbennett, K., Pfannkoch, C., Sumin, N., Sutton, G.G., Viswanathan, L.D., Walenz, B., Goodstein, D.M., Hellsten, U., Kawashima, T., Prochnik, S.E., Putnam, N.H., Shu, S., Blumberg, B., Dana, C.E., Gee, L., Kibler, D.F., Law, L., Lindgens, D., Martinez, D.E., Peng, J., Wigge, P.A., Bertulat, B., Guder, C., Nakamura, Y., Ozbek, S., Watanabe, H., Khalturin, K., Hemmrich, G., Franke, A., Augustin, R., Fraune, S., Hayakawa, E., Hayakawa, S., Hirose, M., Hwang, J.S., Ikeo, K., Nishimiya-Fujisawa, C., Ogura, A., Takahashi, T., Steinmetz, P.R.H., Zhang, X., Aufschnaiter, R., Eder, M.K., Gorny, A.K., Salvenmoser, W., Heimberg, A.M. Wheeler, B.M., Peterson, K.J., Böttger, A., Tischler, P., Wolf, A., Gojobori, T., Remington, K.A., Strausberg, R.L., Venter, J.C., Technau, U., Hobmayer, B., Bosch, T.C.G., Holstein, T.W., Fujisawa, T., Bode, H.R., David, C.N., Rokhsar, D.S., and R.E. Steele. 2010. The dynamic genome of Hydra. Nature. 464:592-596.

Clarke, D.N., Lowe, C.J., and W.J. Nelson. 2019. The cadherincatenin complex is necessary for cell adhesion and embryogenesis in Nematostella vectensis. Developmental Biology. 447:170-181.

Cleves, P.A., Strader, M.E., Bay, L.K., Pringle, J.R., and M.V. Matz. 2018. CRISPR/Cas9-mediated genome editing in a reef-building coral. Proceedings of the National Academy of Sciences. 115:201722151.

Collins, A.G. 2002. Phylogeny of Medusozoa and the evolution of cnidarian life cycles. Journal of Evolutionary Biology. 15:418-432.

Columbus-Shenkar, Y.Y., Sachkova, M.Y., Macrander, J., Fridrich, A., Modepalli, V., Reitzel, A.M., Sunagar, K., and Y. Moran. 2018. Dynamics of venom composition across a complex life cycle. ELife. 7:e35014.

Craggs, J., Guest, J.R., Davis, M., Simmons, J., Dashti, E., and M. Sweet. 2017. Inducing broadcast coral spawning ex situ: Closed system mesocosm design and husbandry protocol. Ecology and Evolution. 7:11066-11078.

Crowell, S. 1946. A new sea anemone from Woods Hole, Massachusetts. Journal of the Washington Academy of Sciences. 36:47-60.

Darling, J.A., Reitzel, A.M., Burton, P.M., Mazza, M.E., Ryan, J.F., Sullivan, J.C., and J.R. Finnerty. 2005. Rising starlet: The starlet sea anemone, Nematostella vectensis. BioEssays. 27:211-221.

Darling, J.A., Reitzel, A.M., and J.R. Finnerty. 2004. Regional population structure of a widely introduced estuarine invertebrate: Nematostella vectensis Stephenson in New England. Molecular Ecology. 13:2969-2981.

DuBuc, T.Q., Stephenson, T.B., Rock, A.Q., and M.Q. Martindale. 2018. Hox and Wnt pattern the primary body axis of an anthozoan cnidarian before gastrulation. Nature Communications. 9:2007.
DuBuc, T.Q., Traylor-Knowles, N., and M.Q. Martindale. 2014. Initiating a regenerative response; cellular and molecular features of wound healing in the cnidarian Nematostella vectensis. BMC Biology. 12:24.

Faltine-Gonzalez, D.Z., and M.J. Layden. 2019. Characterization of NAChRs in Nematostella vectensis supports neuronal and non-neuronal roles in the cnidarian-bilaterian common ancestor. EvoDevo. 10:27.

Fautin, D.G. 2002. Reproduction of Cnidaria. Canadian Journal of Zoology. 80:1735-1754.

Finnerty, J.R., Pang, K., Burton, P., Paulson, D., and M.Q. Martindale. 2004. Origins of bilateral symmetry: Hox and Dpp expression in a sea anemone. Science. 304:1335-1337.

Fischer, A.H.L., Mozzherin, D.A.M.E., Lans, K.D., Wilson, N., Cosentino, C., and J. Smith. 2014. SeaBase: A multispecies transcriptomic resource and platform for gene network inference. Integrative and Comparative Biology. 54:250-263.

Frank, P., and J.S. Bleakney. 1976. Histology and sexual reproduction of the anemone Nematostella vectensis Stephenson 1935. Journal of Natural History. 10:441-449.

Frank, P., and J.S. Bleakney. 1978. Asexual reproduction diet and anomalies of the anemone Nematostella vectensis in Nova Scotia Canada. Canadian Field Naturalist. 259-263.

Fricano, C., Röttinger, E., Furla, P., and S. Barnay-Verdier. 2020. Cnidarian cell cryopreservation: A powerful tool for cultivation and functional assays. Cells. 9:2541.

Friedman, L.E., Gilmore, T.D., and J.R. Finnerty. 2018. Intraspecific variation in oxidative stress tolerance in a model cnidarian: Differences in peroxide sensitivity between and within populations of Nematostella vectensis. PLoS One. 13:e188265.

Fritz, A.E., Ikmi, A., Seidel, C., Paulson, A., and M.C. Gibson. 2013. Mechanisms of tentacle morphogenesis in the sea anemone Nematostella vectensis. Development. 140:2212-2223.

Fritzenwanker, J.H., Genikhovich, G., Kraus, Y., and U. Technau. 2007. Early development and axis specification in the sea anemone Nematostella vectensis. Developmental Biology. 310:264-279.

Fritzenwanker, J.H., and U. Technau. 2002. Induction of gametogenesis in the basal cnidarian Nematostella vectensis (Anthozoa). Development Genes and Evolution. 212:99-103.

Galliot, B., Quiquand, M., Ghila, L., de Rosa, R., Miljkovic-Licina, M., and S. Chera. 2009. Origins of neurogenesis, a Cnidarian view. Developmental Biology. 332:2-24.

Genikhovich, G., and U. Technau. 2009. The starlet sea anemone Nematostella vectensis: An anthozoan model organism for studies in comparative genomics and functional evolutionary developmental biology. Cold Spring Harbor Protocols. 2009:pdb.emo129.

Genikhovich, G., and U. Technau. 2017. On the evolution of bilaterality. Development. 144:3392-3404.

Gosse, P.H. 1860. Actinologia britannica: A history of the British sea-anemones and corals. London: Van Voorst.

Graham, A., Maden, M., and R. Krumlauf. 1991. The murine Hox-2 genes display dynamic dorsoventral patterns of expression during central nervous system development. Development. 112:255-264.

Hand, C. 1957. Another sea anemone from California and the types of certain Californian anemones. Journal of the Washington Academy of Sciences. 47:411-414.

Hand, C., and K.R. Uhlinger. 1992. The culture, sexual and asexual reproduction, and growth of the sea anemone Nematostella vectensis. The Biological Bulletin. 182:169-76.

Hand, C., and K.R. Uhlinger. 1994. The unique, widely distributed, estuarine sea anemone, Nematostella vectensis 
Stephenson: A review, new facts, and questions. Estuaries. 17:501.

Hand, C., and K.R. Uhlinger. 1995. Asexual reproduction by transverse fission and some anomalies in the sea anemone Nematostella vectensis. Invertebrate Biology. 114:9.

Harrison, P.L., Babcock, R.C., Bull, G.D., Oliver, J.K., Wallace, C.C., and B.L. Willis. 1984. Mass spawning in tropical reef corals. Science. 223:1186-1189.

Havrilak, J.A., Al-Shaer, L., Baban, N., Akinci, N., and M.J. Layden. 2021. Characterization of the dynamics and variability of neuronal subtype responses during growth, degrowth, and regeneration. BMC Biology. 19:104.

Havrilak, J.A., Faltine-Gonzalez, D., Wen, Y., Fodera, D., Simpson, A.C., Magie, C.R., and M.J. Layden. 2017. Characterization of NvLWamide-like neurons reveals stereotypy in Nematostella nerve net development. Developmental Biology. 431:336-346.

Havrilak, J.A., and M.J. Layden. 2019. Reverse genetic approaches to investigate the neurobiology of the cnidarian sea anemone Nematostella vectensis. In Methods in molecular biology, ed. S.G. Sprecher, 25-43. New York: Humana Press.

He, S., del Viso, F., Chen, C.Y., Ikmi, A., Kroesen, A.E., and M.C. Gibson. 2018. An axial Hox code controls tissue segmentation and body patterning in Nematostella vectensis. Science. 361:1377-1380.

Hejnol, A., and F. Rentzsch. 2015. Neural nets. Current Biology. 25:R782-R786.

Helm, R.R., Siebert, S., Tulin, S., Smith, J., and C.W. Dunn. 2013. Characterization of differential transcript abundance through time during Nematostella vectensis development. BMC Genomics. 14:266.

Ikmi, A., McKinney, S.A., Delventhal, K.M., and M.C. Gibson. 2014. TALEN and CRISPR/Cas9-mediated genome editing in the early-branching metazoan Nematostella vectensis. Nature Communications. 5:5486.

Ikmi, A., Steenbergen, P.J., Anzo, M., McMullen, M.R. Stokkermans, A., Ellington, L.R., and M.C. Gibson. 2020. Feeding-dependent tentacle development in the sea anemone Nematostella vectensis. Nature Communications. 11:4399.

Jahnel, S.M., Walzl, M., and U. Technau. 2014. Development and epithelial organisation of muscle cells in the sea anemone Nematostella vectensis. Frontiers in Zoology. 11:44.

Karabulut, A., He, S., Chen, C.Y., McKinney, S.A., and M.C. Gibson. 2019. Electroporation of short hairpin RNAs for rapid and efficient gene knockdown in the starlet sea anemone, Nematostella vectensis. Developmental Biology. 448:7-15.

Kass-Simon, G., and A.A. Scappaticci Jr. 2002. The behavioral and developmental physiology of nematocysts. Canadian Journal of Zoology. 80:1772-1794.

Keith, S.A., Maynard, J.A., Edwards, A.J., Guest, J.R., Bauman, A.G., van Hooidonk, R., Heron, S.F., Berumen, M.L., Bouwmeester, J., Piromvaragorn, S., Rahbek C., and A.H. Baird. 2016. Coral mass spawning predicted by rapid seasonal rise in ocean temperature. Proceedings of the Royal Society B: Biological Sciences. 283:20160011.

Kortschak, R.D., Samuel, G., Saint, R., and D.J. Miller. 2003. EST analysis of the cnidarian Acropora millepora reveals extensive gene loss and rapid sequence divergence in the model invertebrates. Current Biology. 13:2190-2195.

Kraus, Y., Aman, A., Technau, U., and G. Genikhovich. 2016. Prebilaterian origin of the blastoporal axial organizer. Nature Communications. 7:11694.

Kraus, Y., and U. Technau. 2006. Gastrulation in the sea anemone Nematostella vectensis occurs by invagination and immigration: An ultrastructural study. Development Genes and Evolution. 216:119-132.

Kumburegama, S., Wijesena, N., Xu, R., and A.H. Wikramanayake. 2011. Strabismus-mediated primary archenteron invagination is uncoupled from Wnt/ $\beta$-catenin-dependent endoderm cell fate specification in Nematostella vectensis (Anthozoa, Cnidaria): Implications for the evolution of gastrulation. EvoDevo. 2:2.

Kusserow, A., Pang, K., Sturm, C., Hrouda, M., Lentfer, J., Schmidt, H.A., Technau, U., von Haeseler, A., Hobmayer, B., Martindale M.Q., and W. Thomas. 2005. Unexpected complexity of the Wnt gene family in a sea anemone. Nature. 433:156-160.

Layden, M.J., Boekhout, M., and M.Q. Martindale. 2012. Nematostella vectensis achaete-scute homolog NvashA regulates embryonic ectodermal neurogenesis and represents an ancient component of the metazoan neural specification pathway. Development. 139:1013-1022.

Layden, M.J., Johnston, H., Amiel, A.R., Havrilak, J., Steinworth, B., Chock, T., Röttinger, E., and M.Q. Martindale. 2016. MAPK signaling is necessary for neurogenesis in Nematostella vectensis. BMC Biology. 14:61.

Layden, M.J., and M.Q. Martindale. 2014. Non-canonical Notch signaling represents an ancestral mechanism to regulate neural differentiation. EvoDevo. 5:30.

Layden, M.J., Rentzsch, F., and E. Röttinger. 2016. The rise of the starlet sea anemone Nematostella vectensis as a model system to investigate development and regeneration. Wiley Interdisciplinary Reviews: Developmental Biology. 5:408-428.

Layden, M.J., Röttinger, E., Wolenski, F.S., Gilmore, T.D., and M.Q. Martindale. 2013. Microinjection of mRNA or morpholinos for reverse genetic analysis in the starlet sea anemone, Nematostella vectensis. Nature Protocols. 8:924-934.

Leclère, L., Bause, M., Sinigaglia, C., Steger, J., and F. Rentzsch. 2016. Development of the aboral domain in Nematostella requires $\beta$-catenin and the opposing activities of Six $3 / 6$ and Frizzled5/8. Development. 143:1766-1777.

Leclère, L., and F. Rentzsch. 2014. RGM regulates BMP-mediated secondary axis formation in the sea anemone Nematostella vectensis. Cell Reports. 9:1921-1930.

Lee, P.N., Kumburegama, S., Marlow, H.Q., M.Q. Martindale, and A.H. Wikramanayake. 2007. Asymmetric developmental potential along the animal: Vegetal axis in the anthozoan cnidarian, Nematostella vectensis, is mediated by Dishevelled. Developmental Biology. 310:169-186.

Magie, C.R., Daly, M., and M.Q. Martindale. 2007. Gastrulation in the cnidarian Nematostella vectensis occurs via invagination not ingression. Developmental Biology. 305:483-497.

Magie, C.R., Pang, K., and M.Q. Martindale. 2005. Genomic inventory and expression of Sox and Fox genes in the cnidarian Nematostella vectensis. Development Genes and Evolution. 215:618-630.

Marlow, H.Q., Matus, D.Q., and M.Q. Martindale. 2013. Ectopic activation of the canonical Wnt signaling pathway affects ectodermal patterning along the primary axis during larval development in the anthozoan Nematostella vectensis. Developmental Biology. 380:324-334.

Marlow, H.Q., Srivastava, M., Matus, D.Q., Rokhsar, D., and M.Q. Martindale. 2009. Anatomy and development of the nervous system of Nematostella vectensis, an anthozoan cnidarian. Developmental Neurobiology. 69:235-254.

Martindale, M.Q., Finnerty, J.R., and J.Q. Henry. 2002. The radiata and the evolutionary origins of the bilaterian body plan. Molecular Phylogenetics and Evolution. 24:358-365. 
Martindale, M.Q., Pang, K., and J.R. Finnerty. 2004. Investigating the origins of triploblasty: 'mesodermal' gene expression in a diploblastic animal, the sea anemone Nematostella vectensis (Phylum, Cnidaria; Class, Anthozoa). Development. 131:2463-2474.

Matus, D.Q., Magie, C.R., Pang, K., Martindale, M.Q., and G.H. Thomsen. 2008. The Hedgehog gene family of the cnidarian, Nematostella vectensis, and implications for understanding metazoan Hedgehog pathway evolution. Developmental Biology. 313:501-518.

Matus, D.Q., Pang, K., Marlow, H.Q., Dunn, C.W., Thomsen, G.H., and M.Q. Martindale. 2006. Molecular evidence for deep evolutionary roots of bilaterality in animal development. Proceedings of the National Academy of Sciences. 103:11195-11200.

Medina, M., Collins, A.G., Silberman, J.D., and M.L. Sogin. 2001. Evaluating hypotheses of basal animal phylogeny using complete sequences of large and small subunit rRNA. Proceedings of the National Academy of Sciences. 98:9707-9712.

Moran, Y., Genikhovich, G., Gordon, D., Wienkoop, S., Zenkert, C., Özbek, S., Technau, U., and M. Gurevitz. 2011. Neurotoxin localization to ectodermal gland cells uncovers an alternative mechanism of venom delivery in sea anemones. Proceedings of the Royal Society B: Biological Sciences. 279:1351-1358.

Nakanishi, N., Renfer, E., Technau, U., and F. Rentzsch. 2012. Nervous systems of the sea anemone Nematostella vectensis are generated by ectoderm and endoderm and shaped by distinct mechanisms. Development. 139:347-357.

Niehrs, C. 2010. On growth and form: A Cartesian coordinate system of Wnt and BMP signaling specifies bilaterian body axes. Development. 137:845-857.

Passamaneck, Y.J., and M.Q. Martindale. 2012. Cell proliferation is necessary for the regeneration of oral structures in the anthozoan cnidarian Nematostella vectensis. BMC Developmental Biology. 12:34.

Pearson, C.V.M., Rogers, A.D., and M. Sheader. 2002. The genetic structure of the rare lagoonal sea anemone, Nematostella vectensis Stephenson (Cnidaria; Anthozoa) in the United Kingdom based on RAPD analysis. Molecular Ecology. 11:2285-2293.

Pearson, J.C., Lemons, D., and W. McGinnis. 2005. Modulating Hox gene functions during animal body patterning. Nature Reviews Genetics. 6:893-904.

Pollock, F.J., Katz, S.M., van de Water, J.A.J.M., Davies, S.W., Hein, M., Torda, G., Matz, M.V., Beltran, V.H., Buerger, P., Puill-Stephan, E., Abrego, D., Bourne, D.G., and B.L. Willis. 2017. Coral larvae for restoration and research: A large-scale method for rearing Acropora millepora larvae, inducing settlement, and establishing symbiosis. PeerJ. 5:e3732.

Posey, M.H., and A.H. Hines. 1991. Complex predator-prey interactions within an estuarine benthic community. Ecology. 72: 2155-2169.

Putnam, N.H., Srivastava, M., Hellsten, U., Dirks, B., Chapman, J., Salamov, A., Terry, A., Shapiro, H., Lindquist, E., Kapitonov, V.V., Jurka, J., Genikhovich, G., Grigoriev, I.V., Lucas, S.M., Steele, R.E., Finnerty, J.R., Technau, U., Martindale, M.Q., and D.S. Rokhsar. 2007. Sea anemone genome reveals ancestral eumetazoan gene repertoire and genomic organization. Science. 317:86-94.

Rabinowitz, C., Moiseeva, E., and B. Rinkevich. 2016. In vitro cultures of ectodermal monolayers from the model sea anemone Nematostella vectensis. Cell and Tissue Research. 366:693-705.
Reitzel, A.M., Burton, P.M., Krone, C., and J.R. Finnerty. 2007. Comparison of developmental trajectories in the starlet sea anemone Nematostella vectensis: Embryogenesis, regeneration, and two forms of asexual fission. Invertebrate Biology. 126:99-112.

Reitzel, A.M., Chu, T., Edquist, S., Genovese, C., Church, C., Tarrant, A.M., and J.R. Finnerty. 2013. Physiological and developmental responses to temperature by the sea anemone Nematostella vectensis. Marine Ecology Progress Series. 484:115-130.

Reitzel, A.M., Darling, J.A., Sullivan, J.C., and J.R. Finnerty. 2007. Global population genetic structure of the starlet anemone Nematostella vectensis: Multiple introductions and implications for conservation policy. Biological Invasions. 10:1197-1213.

Reitzel, A.M., Herrera, S., Layden, M.J., Martindale, M.Q., and T.M. Shank. 2013. Going where traditional markers have not gone before: Utility of and promise for RAD sequencing in marine invertebrate phylogeography and population genomics. Molecular Ecology. 22:2953-2970.

Renfer, E., Amon-Hassenzahl, A., Steinmetz, P.R.H., and U. Technau. 2010. A muscle-specific transgenic reporter line of the sea anemone, Nematostella vectensis. Proceedings of the National Academy of Sciences of the United States of America. 107:104-108.

Renfer, E., and U. Technau. 2017. Meganuclease-assisted generation of stable transgenics in the sea anemone Nematostella vectensis. Nature Protocols. 12:1844-1854.

Rentzsch, F., Anton, R., Saina, M., Hammerschmidt, M., Holstein, T.W., and U. Technau. 2006. Asymmetric expression of the BMP antagonists Chordin and Gremlin in the sea anemone Nematostella vectensis: Implications for the evolution of axial patterning. Developmental Biology. 296:375-387.

Rentzsch, F., Fritzenwanker, J.H., Scholz, C.B., and U. Technau. 2008. FGF signaling controls formation of the apical sensory organ in the cnidarian Nematostella vectensis. Development. 135:1761-1769.

Rentzsch, F., Layden, M.J., and M. Manuel. 2017. The cellular and molecular basis of cnidarian neurogenesis. Wiley Interdisciplinary Reviews: Developmental Biology. 6:e257.

Rentzsch, F., and U. Technau. 2016. Genomics and development of Nematostella vectensis and other anthozoans. Current Opinion in Genetics \& Development. 39:63-70.

Richards, G.S., and F. Rentzsch. 2014. Transgenic analysis of a SoxB gene reveals neural progenitor cells in the cnidarian Nematostella vectensis. Development. 141:4681-4689.

Richards, G.S., and F. Rentzsch. 2015. Regulation of Nematostella neural progenitors by Soxb, Notch and bHLH genes. Development. 142:3332-3342.

Röttinger, E., Dahlin, P., and M.Q. Martindale. 2012. A framework for the establishment of a cnidarian gene regulatory network for 'endomesoderm' specification: The inputs of B-catenin/ TCF signaling. PLoS Genetics. 8:e1003164.

Ryan, J.F., Burton, P.M., Mazza, M.E., Kwong, G.K., Mullikin, J.C., and J.R. Finnerty. 2006. The cnidarian-bilaterian ancestor possessed at least 56 homeoboxes: Evidence from the starlet sea anemone, Nematostella vectensis. Genome Biology. 7:R64.

Saina, M., Genikhovich, G., Renfer, E., and U. Technau. 2009. BMPs and Chordin regulate patterning of the directive axis in a sea anemone. Proceedings of the National Academy of Sciences of the United States of America. 106:18592-18597. Schaffer, A.A., Bazarsky, M., Levy, K., Chalifa-Caspi, V., and U. Gat. 2016. A transcriptional time-course analysis of oral 
vs. aboral whole-body regeneration in the sea anemone Nematostella vectensis. BMC Genomics. 17:718.

Scholz, C.B., and U. Technau. 2003. The ancestral role of Brachyury: Expression of NemBra1 in the basal cnidarian Nematostella vectensis (Anthozoa). Development Genes and Evolution. 212:563-570.

Schwaiger, M., Schönauer, A., Rendeiro, A.F., Pribitzer, C., Schauer, A., Gilles, A.F., Schinko, J.B., Renfer, E., Fredman, D., and U. Technau. 2014. Evolutionary conservation of the eumetazoan gene regulatory landscape. Genome Research. 24:639-650.

Sebé-Pedrós, A., Saudemont, B., Chomsky, E., Plessier, F., Mailhé, M.P., Renno, J., Loe-Mie, Y., Lifshitz A., Mukamel Z., Schmutz S., Novault S., Steinmetz P.R.H., Spitz F., Tanay A., H. Marlow. 2018. Cnidarian cell type diversity and regulation revealed by whole-organism single-cell RNA-seq. Cell. 173:1520-1534.

Servetnick, M.D., Steinworth, B., Babonis, L.S., Simmons, D., Salinas-Saavedra, M., and M.Q. Martindale. 2017. Cas9mediated excision of Nematostella brachyury disrupts endoderm development, pharynx formation, and oral-aboral patterning. Development. 144:dev.145839.

Sheader, M., Suwailem, A.M., and G.A. Rowe. 1997. The anemone, Nematostella vectensis, in Britain: Considerations for conservation management. Aquatic Conservation: Marine and Freshwater Ecosystems. 7:13-25.

Silva, J.F., Lima, C.A.C., Perez, C.D., and P.B. Gomes. 2010. First record of the sea anemone Nematostella vectensis (Actiniaria: Edwardsiidae) in southern hemisphere waters. Zootaxa. 2343:66-68.

Sinigaglia, C., Busengdal, H., Lerner, A., Oliveri, P., and F. Rentzsch. 2015. Molecular characterization of the apical organ of the anthozoan Nematostella vectensis. Developmental Biology. 398:120-133.

Stefanik, D.J., Friedman, L.E., and J.R. Finnerty. 2013. Collecting, rearing, spawning and inducing regeneration of the starlet sea anemone, Nematostella vectensis. Nature Protocols. 8:916-923.

Steinmetz, P.R.H. 2019. A non-bilaterian perspective on the development and evolution of animal digestive systems. Cell and Tissue Research. 377:321-339.

Steinmetz, P.R.H., Aman, A., Kraus, J.E.M., and U. Technau. 2017. Gut-like ectodermal tissue in a sea anemone challenges germ layer homology. Nature Ecology \& Evolution. 1:1535-1542.

Stephenson, T.A. 1935. The British sea anemones. London: Ray Society.

Sunagar, K., Columbus-Shenkar, Y.Y., Fridrich, A., Gutkovich, N., Aharoni, R., and Y. Moran. 2018. Cell type-specific expression profiling unravels the development and evolution of stinging cells in sea anemone. BMC Biology. 16:108.

Szczepanek, S., Cikala, M., and C.N. David. 2002. Poly-gammaglutamate synthesis during formation of nematocyst capsules in Hydra. Journal of Cell Science. 115:745-751.

Takahashi, C.K., Lourenço, N.G.G.S., Lopes, T.F., Rall, V.L.M., and C.A.M. Lopes. 2008. Ballast water: A review of the impact on the world public health. Journal of Venomous Animals and Toxins Including Tropical Diseases. 14:393-408.

Tarrant, A.M., Helm, R.R., Levy, O., and H.E. Rivera. 2019. Environmental entrainment demonstrates natural circadian rhythmicity in the cnidarian Nematostella vectensis. The Journal of Experimental Biology. 222: jeb205393.

Technau, U., and M. Schwaiger. 2015. Recent advances in genomics and transcriptomics of cnidarians. Marine Genomics. 24:31-38.
Technau, U., and R.E. Steele. 2011. Evolutionary crossroads in developmental biology: Cnidaria. Development. 138:14471458 .

Thurm, U., Brinkmann, M., Golz, R., Holtmann, M., Oliver, D., and T. Sieger. 2004. Mechanoreception and synaptic transmission of hydrozoan nematocytes. Hydrobiologia. 530-531:97-105.

Toren, A., and M. Gurovich. 2016. An innovative method to optimize cosmeceutical delivery to skin natural, safe, painless, sustainable transdermal microinjection based on sea anemone nematocysts. Household and Personal Care Today. 11: 41-46.

Torres-Méndez, A., Bonnal, S., Marquez, Y., Roth, J., Iglesias, M., Permanyer, J., Almudí, I., O’Hanlon, D., Guitart, T., Soller, M., Gingras, A.C., Gebauer, F., Rentzsch, F., Blencowe, B.J., Valcárcel, J., and M. Irimia. 2019. A novel protein domain in an ancestral splicing factor drove the evolution of neural microexons. Nature Ecology \& Evolution. 3:691-701.

Trevino, M., Stefanik, D.J., Rodriguez, R., Harmon, S., and P.M. Burton. 2011. Induction of canonical Wnt signaling by alsterpaullone is sufficient for oral tissue fate during regeneration and embryogenesis in Nematostella vectensis. Developmental Dynamics. 240:2673-2679.

Tucker, R.P., Shibata, B., and T.N. Blankenship. 2011. Ultrastructure of the mesoglea of the sea anemone Nematostella vectensis (Edwardsiidae). Invertebrate Biology. 130:11-24.

Tulin, S., Aguiar, D., Istrail, S., and J. Smith. 2013. A quantitative reference transcriptome for Nematostella vectensis early embryonic development: A pipeline for de novo assembly in emerging model systems. EvoDevo. 4:16.

Wainright, P.O., Hinkle, G., Sogin, M.L., and S.K. Stickel. 1993. Monophyletic origins of the metazoa: An evolutionary link with fungi. Science. 260:340-342.

Warner, J.F., Amiel, A.R., Johnston, H., and E. Röttinger. 2019. Regeneration is a partial redeployment of the embryonic gene network. BioRxiv.

Warner, J.F., Guerlais, V., Amiel, A.R., Johnston, H., Nedoncelle, K., and E. Röttinger. 2018. NvERTx: A gene expression database to compare embryogenesis and regeneration in the sea anemone Nematostella vectensis. Development. 145:dev.162867.

Watanabe, H., Fujisawa, T., and T.W. Holstein. 2009. Cnidarians and the evolutionary origin of the nervous system. Development, Growth \& Differentiation. 51:167-183.

Watanabe, H., Kuhn, A., Fushiki, M., Agata, K., Özbek, S., Fujisawa, T., and T.W. Holstein. 2014. Sequential actions of $\beta$-catenin and Bmp pattern the oral nerve net in Nematostella vectensis. Nature Communications. 5:5536.

Weir, K., Dupre, C., van Giesen, L., Lee, A.S.Y., and N.W. Bellono. 2020. A molecular filter for the cnidarian stinging response. eLife. 9:e57578.

Wijesena, N., Simmons, D.K., and M.Q. Martindale. 2017. Antagonistic BMP-cWNT signaling in the cnidarian Nematostella vectensis reveals insight into the evolution of mesoderm. Proceedings of the National Academy of Sciences. 114:E5608-E5615.

Wikramanayake, A.H., Hong, M., Lee, P.N., Pang, K., Byrum, C.A., Bince, J.M., Xu, R., and M.Q. Martindale. 2003. An ancient role for nuclear $\beta$-catenin in the evolution of axial polarity and germ layer segregation. Nature. 426:446-450.

Williams, R.B. 1975. A redescription of the brackish-water sea anemone Nematostella vectensis Stephenson, with an appraisal of congeneric species. Journal of Natural History. 9:51-64. 
Williams, R.B. 1976. Conservation of the sea anemone Nematostella vectensis in Norfolk, England and its world distribution. Transactions of the Norfolk and Norwich Naturalists' Society. 23:257-266.

Williams, R.B. 1979. Studies on the nematosomes of Nematostella vectensis Stephenson (Coelenterata: Actiniaria). Journal of Natural History. 13:69-80.

Williams, R.B. 1983. Starlet sea anemone: Nematostella vectensis. In The IUCN invertebrate red data book, ed. S.M. Wells, R.M. Pyle, and N.M. Collins, 43-46. Gland: IUCAN.

Williams, R.B. 2003. Locomotory behaviour and functional morphology of Nematostella vectensis (Anthozoa: Actiniaria: Edwardsiidae): A contribution to a comparative study of burrowing behaviour in athenarian sea anemones. Zoologische Verhandelingen. 345:437-84.

Wiltse, W.I., Foreman, K.H., Teal, J.M., and I. Valiela. 1984. Effects of predators and food resources on the macrobenthos of salt marsh creeks. Journal of Marine Research. 42:923-942.

Wolenski, F.S., Garbati, M.R., Lubinski, T.J., Traylor-Knowles, N., Dresselhaus, E., Stefanik, D.J., Goucher, H., Finnerty, J.F., and T.D. Gilmore. 2011. Characterization of the core elements of the NF-KB signaling pathway of the sea anemone Nematostella vectensis. Molecular and Cellular Biology. 31:1076-1087.

Wolenski, F.S., Layden, M.J., Martindale, M.Q., Gilmore T.D., and J.R. Finnerty. 2013. Characterizing the spatiotemporal expression of RNAs and proteins in the starlet sea anemone, Nematostella vectensis. Nature Protocols. 8:900-915.

Yonge, M. 1962. Thomas Alan Stephenson, 1898-1961. Biographical Memoirs of Fellows of the Royal Society. 8:136-148.

Zang, H., and N. Nakanishi. 2020. Expression analysis of cnidarian-specific neuropeptides in a sea anemone unveils an apical-organ-associated nerve net that disintegrates at metamorphosis. Frontiers in Endocrinology. 11:63.

Zenkert, C., Takahashi, T., Diesner, M.O., and S. Özbek. 2011. Morphological and molecular analysis of the Nematostella vectensis cnidom. PLoS One. 6:e22725.

Zimmermann, B., Robb, S.M.C., Genikhovich, G., Fropf, W.J., Weilguny, L., He, S., Chen, S., Lovegrove-Walsh, J., Hill, E.M., Ragkousi K., Praher, D., Fredman, D., Moran, Y., Gibson, M.C., and U. Technau. 2020. Sea anemone genomes reveal ancestral metazoan chromosomal macrosynteny. BioRxiv. 